\title{
Optical response from terahertz to visible light of electronuclear transitions in $\mathrm{LiYF}_{4}: \mathrm{Ho}^{3+}$
}

\author{
G. Matmon,,${ }^{1, *}$ S. A. Lynch, ${ }^{2}$ T. F. Rosenbaum, ${ }^{3}$ A. J. Fisher, ${ }^{1}$ and G. Aeppli ${ }^{4}$ \\ ${ }^{1}$ London Centre for Nanotechnology, University College London, 17-19 Gordon Street, London WC1H 0AH, United Kingdom \\ ${ }^{2}$ School of Physics and Astronomy, Cardiff University, Queens Buildings, The Parade, Cardiff CF24 3AA, United Kingdom \\ ${ }^{3}$ Division of Physics, Mathematics, and Astronomy, California Institute of Technology, Pasadena, California 91125, USA \\ ${ }^{4}$ Institute of Physics, Ecole Polytechnique Fédérale de Lausanne (EPFL), 1015 Lausanne, Department of Physics, ETH Zürich, 8093 Zürich, \\ and Paul Scherrer Institute, 5232 Villigen, Switzerland
}

(Received 4 May 2016; revised manuscript received 18 October 2016; published 21 November 2016; corrected 2 December 2016)

\begin{abstract}
Because of its role as a model system with tunable quantum fluctuations and quenched disorder, and the desire for optical control and readout of its states, we have used high-resolution optical absorption spectroscopy to measure the crystal-field excitations for $\mathrm{Ho}^{3+}$ ions in $\mathrm{LiHo}_{x} \mathrm{Y}_{1-x} \mathrm{~F}_{4}$ from the terahertz to visible regimes. We show that many of the excitations yield very narrow lines visibly split even by the nuclear hyperfine interaction, making $\mathrm{Ho}^{3+}$ in $\mathrm{LiHo}_{x} \mathrm{Y}_{1-x} \mathrm{~F}_{4}$ a candidate host for optically addressable electronuclear qubits with quality factors as high as $Q=4.7 \times 10^{5}$, where the higher-lying levels are electronic singlets. Optical transitions in the easily accessible near- and mid-infrared are narrow enough to allow readout of the ground-state electronuclear qubits responsible for the interesting magnetism of $\mathrm{LiHo}_{x} \mathrm{Y}_{1-x} \mathrm{~F}_{4}$. While many of the higher-lying states have been observed previously, we also report here detailed spectra of terahertz excitations. The strengths of the electric and magnetic dipole crystal-field transition lines of five of the lowest excited spin-orbit manifolds of dilute $\mathrm{LiYF}_{4}: \mathrm{Ho}^{3+}$ were calculated and compared with measurement. The magnitude of the nuclear hyperfine coupling was used to assign the correct upper and lower states to transition lines.
\end{abstract}

DOI: 10.1103/PhysRevB.94.205132

\section{INTRODUCTION}

The scheelite crystal $\mathrm{LiY}_{1-x} \mathrm{~F}_{4}: \mathrm{Ho}_{x}^{3+}$ [Fig. 1(a)] has been used in many key demonstrations of collective quantum phenomena in the solid state, including quantum phase transitions [1,2], quantum annealing [3,4], long-lived coherent oscillations [5], and long-range entanglement [6,7]. The magnetic moment of the $\mathrm{Ho}^{3+}$ ions is carried by $f$ electrons which are well isolated from the environment, interacting only by dipolar couplings. This leads to a well-characterized spin-spin interaction which can be controlled by dilution; the characteristic dipolar temperature scale is approximately $1 \mathrm{~K}$ at $x=1$. All this well-studied physics occurs within the lowest $(J=8)$ level derived from the $L=6, S=2$ term of the $f^{10}$ configuration; its states are further split by the crystal field from the surroundings with an energy scale up to $300 \mathrm{~cm}^{-1}$ ( $430 \mathrm{~K}$, i.e., in the $\mathrm{THz}$ region), and these splittings can be probed by neutron scattering [2] or electron-paramagneticresonance (EPR) [8] experiments. The large hyperfine interaction strongly couples the $I=7 / 2$ nuclear spins to the electron spins, resulting in further transitions in the microwave region.

A sequence of higher levels $(J=7, J=6$, etc.) exists at higher energies (split by spin-orbit coupling on the scale $3000-5000 \mathrm{~cm}^{-1}$ and extending through NIR/visible/UV). The energy scales are summarized in Fig. 1(b). These levels are very long-lived, since the optical transitions are very weakly dipole-allowed. The long lifetimes and the correspondingly narrow linewidths $\left(\sim 0.04 \mathrm{~cm}^{-1} \mathrm{FWHM}\right)$ of some of the excited levels make the material suitable as a laser gain medium in the $2 \mu \mathrm{m}$ region, often codoped with $\mathrm{Tm}^{3+}$ (Refs. [9-11]).

The combination of interesting quantum magnetic behavior and rich optical physics makes $\mathrm{LiYF}_{4}: \mathrm{Ho}^{3+}$ a candidate for

\footnotetext{
*g.matmon@ucl.ac.uk
}

studying nonequilibrium quantum mechanics using coherent optical manipulation, and ultimately for quantum information processing. The dipolar interactions between $\mathrm{Ho}^{3+}$ ions confer an important advantage over other point defect systems (e.g., the well-known NV center in diamond), particularly if they can be switched by exploiting the different symmetries of the excited states to control the spins optically. It should be noted that such optomagnetic control of spins would not rely on spin polarization via circularly polarized light as traditionally used in conventional semiconductors [12] but instead on the different intrinsic spins associated with different symmetries of the excited states.

The energy levels of the $\mathrm{LiYF}_{4}: \mathrm{Ho}^{3+}$ system have been studied extensively via a variety of spectroscopies. The crystalfield absorption lines were measured, and their positions calculated by Karayianis et al. [13] and Christensen [14]. The lowest-lying states were also determined by EPR measurements [15] and neutron scattering [2]. Walsh et al. used absorption and high-photon-energy luminescence to extract the absorption and emission cross sections, as well as the decay branching ratios and the radiative lifetimes between manifolds [16]. Spectroscopic measurements of the spin-orbit manifolds have been successfully compared with line-strength calculations using the Judd-Ofeldt theory [16,17], although no account has as yet been given to the intensity distribution of the crystal-field-split lines within the manifolds. High-resolution spectroscopic measurements of $\mathrm{LiY}_{1-x} \mathrm{Ho}_{x} \mathrm{~F}_{4}$, with $x$ in the range 0.001-0.01 [18-20], have shown optically detected nuclear hyperfine interactions, an example of which appears in Fig. 1(b). The energy level configurations leading to some of the observed hyperfine lines were described [18], as well as the effects of $\mathrm{Ho}^{3+}$ pairs [20] and host crystal isotopes [19]. The effects of neighboring pairs with differently oriented separation vectors on the ground-state magnetic properties were studied in [21]. 


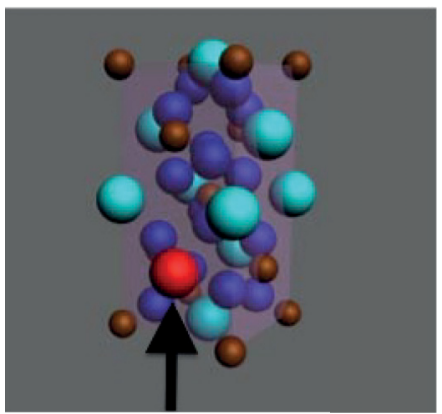

(a)

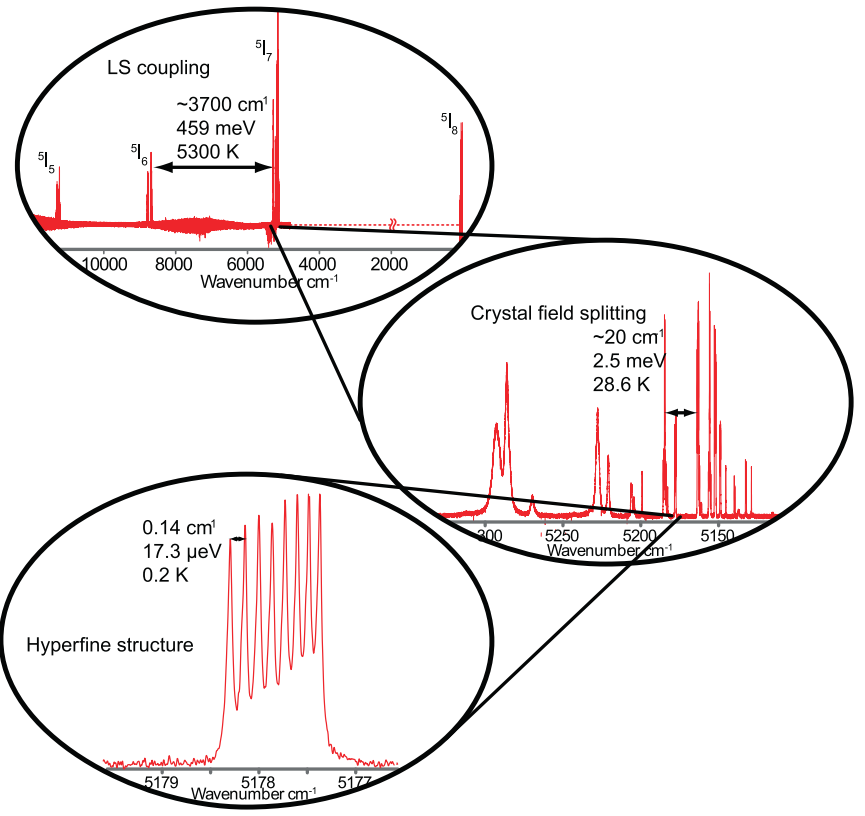

(b)

FIG. 1. The $\mathrm{LiYF}_{4}: \mathrm{Ho}^{3+}$ crystal. (a) The tetragonal unit cell. The light-colored (cyan) spheres represent yttrium atoms; the arrow points to a single holmium atom. (b) Energy scales of holmium electronic transitions. Top: LS coupling; center: crystal-field splitting; bottom: hyperfine interaction.

In this work we report high-resolution, polarizationresolved absorption spectra of $\mathrm{LiY}_{1-x} \mathrm{Ho}_{x} \mathrm{~F}_{4}(x=0.01,0.003)$ in the energy range $20-15700 \mathrm{~cm}^{-1}$, covering the ${ }^{5} I_{8,7,6,5}$ and ${ }^{5} F_{5}$ manifolds. A summary of the experimentally measured lines appears in Fig. 2. We measure the intensities and (where possible) hyperfine splittings, and compare them to the calculated line strengths of individual crystal-field levels. Hence we characterize fully the Hamiltonian of the $\mathrm{Ho}^{3+}$ ion in its crystal environment, its interactions with radiation, and the most important interactions with vibrations of the surrounding lattice. We measure the temperature-dependent line broadening from $4 \mathrm{~K}$ to $40 \mathrm{~K}$ and suggest its origins.

\section{EXPERIMENTAL AND THEORETICAL METHODS}

\section{A. Optical measurement of high-resolution spectra}

High-resolution $\left(0.01 \mathrm{~cm}^{-1}\right)$ polarized absorption spectra of $\mathrm{LiY}_{1-x} \mathrm{Ho}_{x} \mathrm{~F}_{4}(x=0.01,0.003)$ were collected as a function of temperature $(4-40 \mathrm{~K})$ in the visible and nearand mid-infrared (4000 to $15700 \mathrm{~cm}^{-1}$ ), as well as the

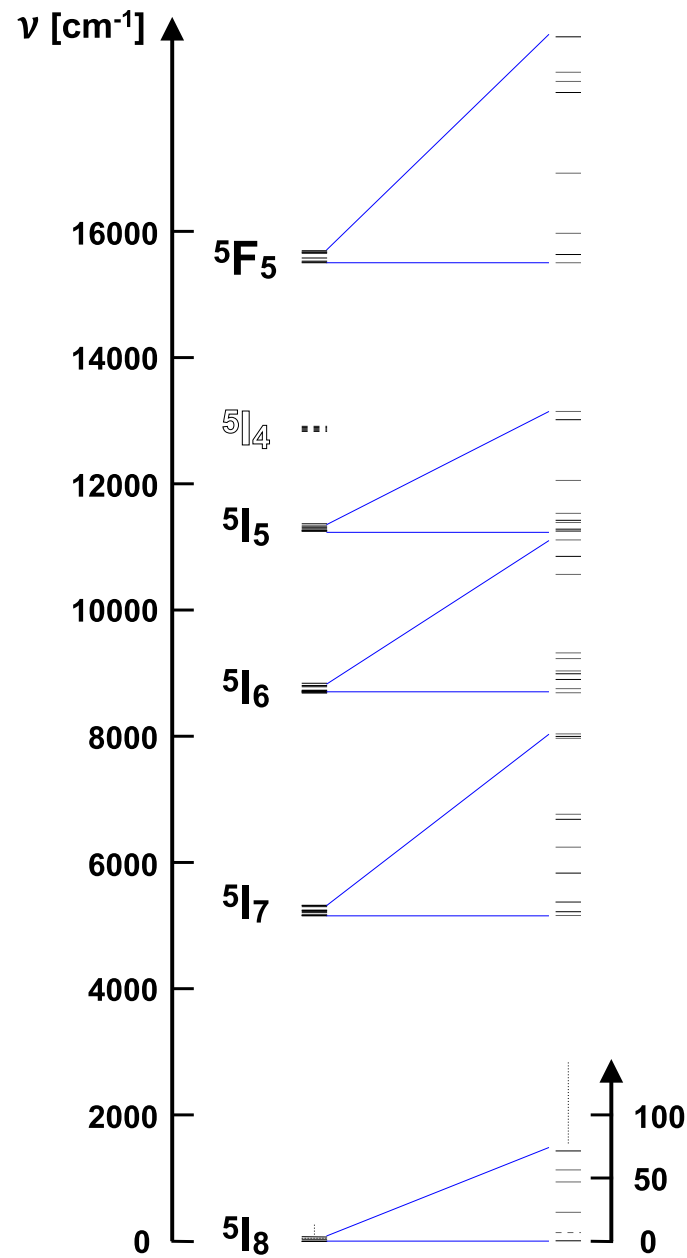

FIG. 2. The energy levels measured in this work. The blowups in the right columns are to scale within each spin-orbit manifold. In the right column gray lines are singlet states and bold lines are doublet states. Dashed lines are known lines that were not measured, including the ${ }^{5} I_{4}$ manifold. The vertical scale at the bottom right is the energy scale of the zoomed-in manifolds, in $\mathrm{cm}^{-1}$.

far-infrared/THz $\left(20-400 \mathrm{~cm}^{-1}\right)$ regimes. The latter spectral region has not, to our knowledge, been optically measured previously in this material.

Fourier transform infrared (FT-IR) absorption spectroscopy was performed using a Bruker $125 \mathrm{HR}$ high-resolution FTIR vacuum spectrometer. The sample was mounted on the cold finger of an Oxford Instruments helium flow cryostat, whose temperature was controlled by a combination of flow control and a heating resistor. A high-pressure mercury source and helium-cooled composite silicon bolometer were used for the far-infrared, and for visible, near-, and mid-infrared measurements a halogen lamp was paired with either a silicon photodiode or a liquid-nitrogen-cooled MCT photoconductive detector. A polarizer was placed between the sample and the detector (wire grid for far-infrared, Glan-Laser otherwise). The sample was oriented such that the FT-IR beam was perpendicular to the $a c$ plane of the crystal, where $c$ is the extraordinary axis. For consistency we will refer to both the crystal axes $a$ and $c$ and the polarization of the fields parallel to them ( $x$ and $z$, respectively) as $x$ and $z$. 


\section{B. Hierarchy of energy scales}

\section{Atomic configurations and the crystal field}

The states concerned differ only in the arrangement of the $f$ electrons in the $\mathrm{Ho}^{3+}$ ions. The $(4 f)^{10}$ configuration is split by the electron-electron Coulomb interaction into terms of different total orbital angular momentum $L$ and total spin angular momentum $S$, of which the lowest has (according to Hund's rules) the maximum possible values $S=2$ and $L=6$. Each term is further split by spin-orbit coupling into levels of different total angular momentum $J$, giving a ground-state level with $J=8$ and excited states $J=7$, $J=6$ and so on. In the solid-state environment the states of each term are then split by the crystal field (incorporating the electrostatic effects of neighboring ions and hybridization with their electronic states); according to the Wigner-Eckart theorem this interaction can be written within each level in terms of the matrix elements of the total angular momentum operator $\hat{\mathbf{J}}$. Although the underlying couplings are the same in terms of the electrons' spatial coordinates, the representation in terms of $\hat{\mathbf{J}}$ differs for each level. The crystal field can then be decomposed into parts $\hat{O}_{l}^{(m)}$ transforming in the same way as the spherical harmonics $Y_{l}^{m}(\theta, \phi)$.

Within the lowest manifold where all states have the same electronic angular momentum $J=8$, the operators $\hat{O}_{l}^{(m)}$ can be taken to be functions of the components of $\hat{\mathbf{J}}[22,23]$ and hence the crystal-field Hamiltonian can be written

$$
\hat{H}_{\mathrm{cf}}=\sum_{l m} B_{l}^{m} \hat{O}_{l}^{(m)}(\mathbf{J})
$$

For $m \neq 0$ it is conventional to reexpress the operators $\hat{O}_{l}^{( \pm m)}$ in terms of the combinations

$$
\begin{aligned}
& \hat{O}_{l}^{m}(c)=\frac{1}{\sqrt{2}}\left[\hat{O}_{l}^{-m}+(-1)^{m} \hat{O}_{l}^{m}\right], \\
& \hat{O}_{l}^{m}(s)=\frac{i}{\sqrt{2}}\left[\hat{O}_{l}^{-m}-(-1)^{m} \hat{O}_{l}^{m}\right],
\end{aligned}
$$

where the designations $c$ and $s$ are used because these operators transform under rotations about the principal (in this case fourfold) axis like $\cos (m \phi)$ and $\sin (m \phi)$, respectively.

Since the crystal-field energies are much smaller than the intra-ion spin-orbit coupling and Coulomb energy, the effect of the crystal-field terms in mixing different configurations is negligible (except in the calculation of electric dipole matrix elements; see below). The point-group symmetry of the Ho site is $S_{4}(4 / \mathrm{m})$; the presence of the fourfold axis constrains the nonzero crystal-field terms $B_{l}^{m}$ to have $m=0$ or $m= \pm 4$, while even though the point group does not contain the inversion operation the odd-parity (odd- $l$ ) terms in the expansion have no effect within the even-parity $(4 f)^{10}$ configuration and can be neglected in the computation of the crystal-field states. This leaves just seven independent terms: $l=2$ and $m=0 ; l=4$ and $m=0, \pm 4 ; l=6$ and $m=0, \pm 4$. For the values of these remaining coefficients we follow Ref. [2] [this implies choosing the axes of the crystal-field Hamiltonian so $B_{4}^{4}$ is real and hence $\left.B_{4}^{4}(s)=0\right]$; these values were derived by fitting to neutron-scattering measurements of the dispersion of excitations within the
TABLE I. Parameters (in meV) appearing in the single-ion Hamiltonian equation (11). Values are taken from Ref. [2].

\begin{tabular}{cccccc}
\hline \hline$B_{2}^{0}$ & $10^{3} B_{4}^{0}$ & $10^{3} B_{4}^{4}(c)$ & $10^{5} B_{6}^{0}$ & $10^{5} B_{6}^{4}(c)$ & $10^{5} B_{6}^{4}(s)$ \\
\hline-0.06 & 0.35 & 3.6 & 0.04 & 7.0 & 0.98 \\
\hline \hline
\end{tabular}

lowest $(J=8)$ level, and to the high-temperature magnetic susceptibility. Hence we have

$$
\begin{aligned}
\hat{H}_{\mathrm{cf}}= & B_{2}^{0} \hat{O}_{2}^{(0)}(\mathbf{J})+\left[B_{4}^{0} \hat{O}_{4}^{(0)}(\mathbf{J})+B_{4}^{4}(c) \hat{O}_{4}^{(4)}(c)(\mathbf{J})\right] \\
& +\left[B_{6}^{0} \hat{O}_{6}^{(0)}(\mathbf{J})+B_{6}^{4}(c) \hat{O}_{6}^{(4)}(c)(\mathbf{J})+B_{6}^{4}(s) \hat{O}_{6}^{(4)}(s)(\mathbf{J})\right] .
\end{aligned}
$$

Although fitted entirely to the ground-state manifold, the Hamiltonian can be applied also to higher levels derived from the $L=6$ term by expressing the crystal field in terms of the orbital angular momentum $\hat{L}$ and then reexpressing this in terms of $\hat{J}$ for each level in turn. The result is

$$
\begin{aligned}
\hat{H}_{\mathrm{cf}}= & \alpha B_{2}^{0} \hat{O}_{2}^{(0)}(\mathbf{L})+\beta\left[B_{4}^{0} \hat{O}_{4}^{(0)}(\mathbf{L})+B_{4}^{4}(c) \hat{O}_{4}^{(4)}(c)(\mathbf{L})\right] \\
& +\gamma\left[B_{6}^{0} \hat{O}_{6}^{(0)}(\mathbf{L})+B_{6}^{4}(c) \hat{O}_{6}^{(4)}(c)(\mathbf{L})+B_{6}^{4}(s) \hat{O}_{6}^{(4)}(s)(\mathbf{L})\right],
\end{aligned}
$$

where $\alpha, \beta$, and $\gamma$ are determined by the ratios of the the reduced matrix elements of the $\hat{\mathbf{L}}$ and $\hat{\mathbf{J}}$ operators: for example,

$$
\alpha=\frac{\left\langle S=2, L=6, J=8\left\|\hat{O}^{(2)}(\mathbf{J})\right\| S=2, L=6, J=8\right\rangle}{\left\langle S=2, L=6\left\|\hat{O}^{(2)}(\mathbf{L})\right\| S=2, L=6\right\rangle} .
$$

For other values of $L$ (for example for the ${ }^{5} F_{5}$ manifold) a similar procedure is followed except that the crystal field is reexpressed in terms of the single-electron orbital angular momentum $\mathbf{l}$.

The values used are shown in Table I.

\section{Hyperfine splittings}

Finally the hyperfine interaction between each electron and the nuclear moment $\left({ }^{165} \mathrm{Ho}\right.$ has nuclear angular momentum $I=7 / 2$ ) can be written in the form $A_{J} \hat{\mathbf{J}} \cdot \hat{\mathbf{I}}$, where $A_{J}$ is the effective hyperfine interaction for angular momentum $J$ :

$$
A_{J}=\frac{a_{f}\left\langle J|| \sum_{i=1}^{N} \hat{\mathbf{N}}_{i}|| J\right\rangle}{\sqrt{J(J+1)(2 J+1)}}
$$

with $a_{f}$ equal to the hyperfine coupling of a single $f$ electron and the reduced matrix element in the numerator is of the operator

$$
\hat{\mathbf{N}}_{i}=\mathbf{l}_{i}-\mathbf{s}_{i}+\frac{3 \mathbf{r}_{i}\left(\mathbf{s}_{i} \cdot \mathbf{r}_{i}\right)}{r_{i}^{2}},
$$

which determines the effective magnetic field of the $i$ th electron at the nuclear site, where $r_{i}$ is the position vector of electron $i$ relative to the nucleus.

Each of the electronic states of a single ion is therefore split into 8 by the interaction with the nuclear spin $I=7 / 2$, but the magnitude of the hyperfine splitting varies enormously for two reasons. First, the coupling between the electronic angular 
TABLE II. Summary of selection rules for electric and magnetic dipole transitions. Each table entry shows which component of the relevant operator can couple states of the given symmetries; for electric and magnetic dipoles the Cartesian component of the corresponding field is given, while for the electric quadrupole two Cartesian components corresponding to a field direction and a gradient direction are given.

\begin{tabular}{|c|c|c|c|c|c|c|c|c|c|c|c|c|}
\hline & \multicolumn{4}{|c|}{ Electric dipole } & \multicolumn{4}{|c|}{ Magnetic dipole } & \multicolumn{4}{|c|}{ Electric quadrupole } \\
\hline & $\Gamma_{1}$ & $\Gamma_{2}$ & $\Gamma_{x}$ & $\Gamma_{y}$ & $\Gamma_{1}$ & $\Gamma_{2}$ & $\Gamma_{x}$ & $\Gamma_{y}$ & $\Gamma_{1}$ & $\Gamma_{2}$ & $\Gamma_{x}$ & $\Gamma_{y}$ \\
\hline$\Gamma_{1}$ & & $z$ & $x$ & $y$ & $z$ & & $x$ & $y$ & $z^{2}$ & $\begin{array}{c}x^{2}-y^{2} \\
x y\end{array}$ & $x z$ & $y z$ \\
\hline$\Gamma_{2}$ & $z$ & & $y$ & $x$ & & $z$ & $y$ & $x$ & $\begin{array}{c}x^{2}-y^{2} \\
x y\end{array}$ & $z^{2}$ & $y z$ & $x z$ \\
\hline$\Gamma_{x}$ & $x$ & $y$ & & $z$ & $x$ & $y$ & $z$ & & $x z$ & $y z$ & $z^{2}$ & $\begin{array}{c}x^{2}-y^{2} \\
x y\end{array}$ \\
\hline$\Gamma_{y}$ & $y$ & $x$ & $z$ & & $y$ & $x$ & & $z$ & $y z$ & $x z$ & $\begin{array}{c}x^{2}-y^{2} \\
x y\end{array}$ & $z^{2}$ \\
\hline
\end{tabular}

momentum $\hat{\mathbf{J}}$ and the nuclear angular momentum $\hat{\mathbf{I}}$ varies with $J$ according to Eq. (7). For the lowest $(J=8)$ manifold, spin-relaxation measurements yield $A_{8} / k_{B}=38.63 \mathrm{mK}$ [24], hence $a_{f} / k_{B}=50.9 \mathrm{mK}$ or $a_{f} /(h c)=0.035 \mathrm{~cm}^{-1}$.

Second, within first-order perturbation theory the nucleus experiences an effective magnetic field proportional to $A\langle\hat{\mathbf{J}}\rangle$, where the expectation value is taken over the electronic state concerned; these expectation values vary greatly. For the $\Gamma_{34}$ states, a nonzero expectation value $\left\langle\hat{J}_{z}\right\rangle$ is allowed; each such electronic state is therefore split into an octet, each state having a different value of $\left\langle\hat{I}_{z}\right\rangle$ [21]. Hence there is a first-order hyperfine shift,

$$
\delta_{M_{I}}^{(1)}=A_{J}\left\langle\hat{J}_{z}\right\rangle M_{I},
$$

with an equal level spacing of $A_{J}\left\langle\hat{J}_{z}\right\rangle$. The values of $\left\langle\hat{J}_{z}\right\rangle$ are equal and opposite in the two components of each $\Gamma_{34}$ doublet, so the order of the nuclear sublevels is reversed. The situation is further complicated because the expectation values $\left\langle\hat{J}_{z}\right\rangle$ for a given doublet can be of either sign. For the ground state $\left\langle\hat{J}_{z}\right\rangle<0$ for the $\Gamma_{3}$ component (see Sec. II C 1 below) [18]; hence the hyperfine splitting of the ground state is $-0.146 \mathrm{~cm}^{-1}$. For absorptions to excited $\Gamma_{34}$ states, the observed line spacings depend on the hyperfine splittings of both levels. To a good approximation the nuclear moment is conserved in the transition; for electric dipole (ED) transitions each $\Gamma_{34}$ component is coupled to the opposite component (see Table II); the inversion of the nuclear levels in the different components of the electronic doublet therefore means that the observed line spacing is the sum of the individual hyperfine splittings $\left(A_{g}\left\langle\hat{J}_{z}\right\rangle_{g}+A_{e}\left\langle\hat{J}_{z}\right\rangle_{e}\right)$ (where $A_{g}$ and $A_{e}$ are the values of $A_{J}$ for the ground- and excitedstate manifolds, respectively). For magnetic dipole (MD) transitions the magnetic field couples each component of the $\Gamma_{34}$ doublet to its partner of the same symmetry, and therefore the observed line spacing is the absolute difference of the individual splittings $\left|A_{g}\left\langle\hat{J}_{z}\right\rangle_{g}-A_{e}\left\langle\hat{J}_{z}\right\rangle_{e}\right|$. Note that since they are proportional to the $z$ projections of angular momentum, the first-order hyperfine splittings for the $\Gamma_{3}\left(\Gamma_{4}\right)$ states in a manifold with $J=8$ or $J=7$ are constrained to sum to $(-7-3+1+5) A_{J}=-4 A_{J}\left(+4 A_{J}\right)$, while for $J=6$ or $J=5$ the total is $(-3+1+5) A_{J}=+3 A_{J}\left(-3 A_{J}\right)$.

For states with $\Gamma_{1}$ or $\Gamma_{2}$ symmetry, on the other hand, $\left\langle\hat{J}_{z}\right\rangle$ is constrained to be zero, so there is no linear hyperfine splitting and the leading term is of quadratic order in $A$. Under most circumstances this is small; for ground-state absorptions to most $\Gamma_{1}$ and $\Gamma_{2}$ states, we will therefore observe a ladder of lines spaced by $0.146 \mathrm{~cm}^{-1}$. However if the ratio of the electronic energy spacings $\Delta E_{j i}$ between nearby excited states of the same symmetry to the hyperfine interaction $A_{e}$ in the excited manifold is not too large, the corresponding secondorder hyperfine shifts in state $i$ will be

$$
\delta_{M_{I}}^{(2)}=A_{e}^{2} \sum_{j} \frac{\left|\left\langle i\left|\hat{J}_{z}\right| j\right\rangle\right|^{2}}{\Delta E_{j i}} M_{I}^{2} \equiv \beta M_{I}^{2},
$$

where $\Delta E_{j i}=E_{j}-E_{i}$, and $M_{I}$ is the magnetic quantum number for the nuclear spin. The spacing between adjacent hyperfine lines is now $\beta\left(2 M_{I}-1\right)-A_{g}\left\langle\hat{J}_{z}\right\rangle_{g}$, and so varies linearly through the octet as shown in Fig. 3(b). Experimentally this frequently manifests itself in an asymmetric line shape when the different hyperfine components overlap.

For absorption from the first excited $\Gamma_{2}$ state, by contrast, there is negligible hyperfine splitting in the lower state and the transition energies therefore reflect only the hyperfine splitting of the upper states.

Figure 3 shows examples of the different hyperfine splittings, selection rules, and level repulsions.

Assembling all these terms, the effective Hamiltonian for the ${ }^{5} L_{J}$ term of an isolated $\mathrm{Ho}^{3+}$ ion within the crystal becomes

$$
\hat{H}_{\text {ion }}=\xi \hat{\mathbf{L}} \cdot \mathbf{S}+\hat{H}_{c f}+A_{J} \hat{\mathbf{J}} \cdot \hat{\mathbf{I}}
$$

where $\xi$ is the atomic spin-orbit coupling.

\section{Optical matrix elements and selection rules}

Since all the states are derived from the $(4 f)^{10}$ configuration of the $\mathrm{Ho}^{3+}$ ion, they have even parity. Hence electric dipole transitions between them are parity-forbidden in the free ion. This leaves three principal sources of coupling to electromagnetic radiation: the magnetic dipole interaction, the electric quadrupole interaction, and weakly allowed electric dipole transitions mediated by the small admixtures of higher oddparity configurations into the even-parity manifolds resulting from the solid-state environment. 


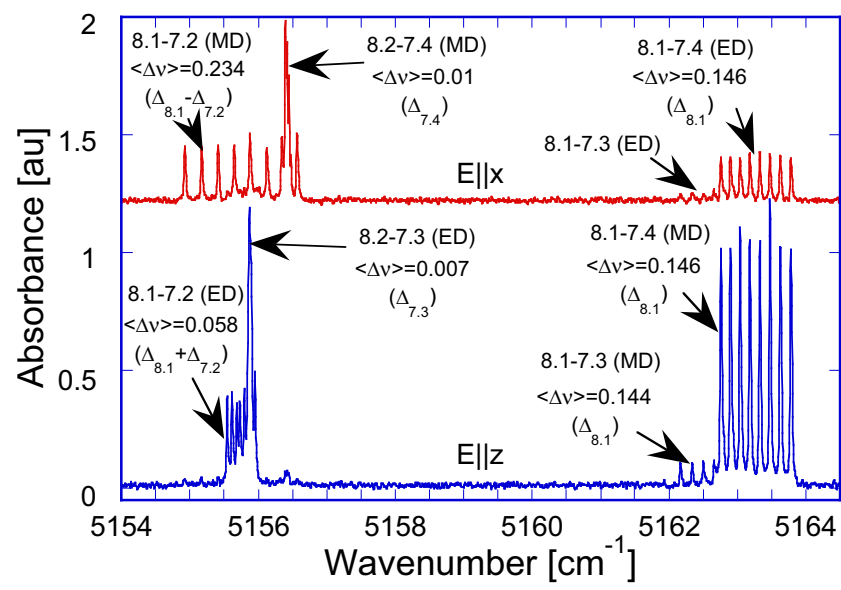

(a)

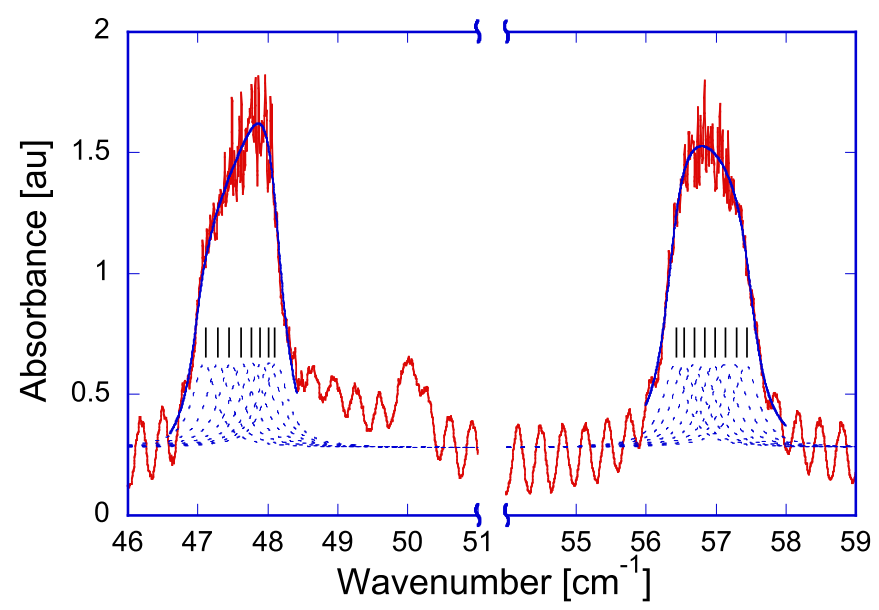

(b)

FIG. 3. Possible lineshapes for transitions of different types and between states of different symmetries. (a) A selection of ED and MD transitions between $\Gamma_{1}, \Gamma_{2}$, and $\Gamma_{34}$ states at $x$ and $z$ (red/top and blue/bottom, respectively) polarizations, demonstrating state assignments through sums and differences of hyperfine splittings. For the state designations see Table $\mathrm{V}$. The $\mathrm{Ho}^{3+}$ concentration is $0.3 \%$, and the nominal temperature is $4 \mathrm{~K}$. (b) An example of the interaction between neighboring levels of the same symmetry [see Eq. (10)], in this case the $\Gamma_{1}$ states 8.4 and 8.5 (see Table IV). The experimental data are in red. The fits and their constituent Lorentzians are in solid and dashed blue, respectively. The vertical black lines indicate the individual hyperfine peak locations. The $\mathrm{Ho}^{3+}$ concentration is $1 \%$, and the nominal temperature is $3.8 \mathrm{~K}$. Fabry-Perot fringes dominate in this spectral region due to decreased effect of the wedge angle at long wavelengths, as well as the higher refractive index. The fringes do not affect areas of strong absorbance, i.e., the transition peaks themselves.

\section{Selection rules}

The point-group symmetry of the Ho site is $S_{4}(\overline{4})$, generated by the combination of a fourfold rotation about the $c$ axis and inversion through the origin (however the simple inversion operation is absent). There are three irreducible representations, two one-dimensional $\left(A, B\right.$ or $\left.\Gamma_{1}, \Gamma_{2}\right)$ and one two-dimensional $\left(E\right.$ or $\left.\Gamma_{34}\right)$. We adopt the convention that $\Gamma_{3}$ and $\Gamma_{4}$ are related to $E_{x}$ and $E_{y}$ in the same way that components of rank-1 spherical tensors are related to the Cartesian coordinates, so $E_{x}=\frac{\Gamma_{4}-\Gamma_{3}}{\sqrt{2}} \equiv \Gamma_{x}$ and $E_{y}=$ $i \frac{\Gamma_{4}+\Gamma_{3}}{\sqrt{2}} \equiv \Gamma_{y}$. We adopt the solid-state $\Gamma$ convention in the remainder of the paper.

The selection rules for magnetic and electric dipole transitions are similar for fields in the $(x, y)$ plane: both pairs of fields transform as $\Gamma_{1}$ and therefore connect $\Gamma_{1}$ and $\Gamma_{2}$ states to $\Gamma_{x}$ and $\Gamma_{y}$ states, respectively. However for fields along the $z$ axis the selection rules are different; the electric dipole operator transforms like $\Gamma_{2}$ and couples $\Gamma_{1}$ to $\Gamma_{2}$ and $\Gamma_{x}$ to $\Gamma_{y}$, while the magnetic dipole operator transforms as $\Gamma_{1}$ and connects $\Gamma_{1}$ to $\Gamma_{1}, \Gamma_{2}$ to $\Gamma_{2}, \Gamma_{x}$ to $\Gamma_{x}$, and $\Gamma_{y}$ to $\Gamma_{y}$. For radiation propagating in the $(x, y)$ plane, there are two independent linear polarizations: if the electric field is in the plane then it couples the ground state to the $\Gamma_{1}$ and $\Gamma_{2}$ states, while the magnetic field lies along the $z$ axis and couples it to $\Gamma_{34}$ states. If the electric field is along the $z$ axis it couples the ground state to $\Gamma_{34}$ states, while the magnetic field couples it to $\Gamma_{1}$ and $\Gamma_{2}$ states. For radiation propagating in the $(x, y)$ plane the selection rules are reversed for the electric quadrupole as compared to electric dipole: for example for radiation propagating along $y$, the $x$ polarization produces the $x y$ quadrupole component which has similar couplings to the $z$ component of the electric field, while the $z$ polarization produces the $y z$ quadrupole which has the same selection rules as the $x$ component of electric field. The selection rules are summarized in Table II.

\section{Magnetic dipole terms}

The magnetic dipole matrix element between electronic crystal-field states $\left|i, J_{i}\right\rangle$ and $\left|j, J_{j}\right\rangle$ in manifolds with total angular momentum $J_{i}, J_{j}$ is

$$
\begin{aligned}
\frac{\mu_{B}}{\hbar} & \mathbf{B} \cdot\left\langle i, J_{i}\left|\hat{\mathbf{L}}+g_{f} \hat{\mathbf{S}}\right| j, J_{j}\right\rangle \\
& \approx \frac{\mu_{B}}{\hbar} \mathbf{B} \cdot\left[2\left\langle i, J_{i}|\hat{\mathbf{J}}| j, J_{j}\right\rangle \delta_{J_{i}, J_{j}}-\left\langle i, J_{i}|\hat{\mathbf{L}}| j, J_{j}\right\rangle\right] \\
& \equiv \mu_{B} \mathbf{B} \cdot \hat{\mathbf{O}}^{\mathrm{MD}},
\end{aligned}
$$

where $g_{f} \approx 2$ is the $g$ factor of a free electron. Since $\hat{\mathbf{L}}$ is a tensor operator with $l=1$, it follows that the selection rule $\Delta J=0, \pm 1$ applies (assuming mixing of different manifolds can be neglected) and therefore the ground $(J=8)$ manifold has nonzero matrix elements connecting it only with the $J=7$ manifold. Note that (neglecting interlevel mixing) the magnetic dipole matrix elements are entirely determined by fundamental constants, angular momentum algebra, and the nature of the individual crystal-field states; once the even-order crystal-field coefficients are given, no further fitting is required in order to extract them.

There is in principle a second source of magnetic-dipole interaction through the coupling of the $\mathbf{B}$ fields to nuclear moments; however this is smaller than the electronic matrix element by a factor $\mu_{N} / \mu_{B} \sim 10^{-3}$ (where $\mu_{N}$ is the nuclear magneton) and we therefore neglect it.

\section{Electric quadrupole terms}

Like magnetic dipole terms, electric quadrupole interactions have even parity and so can couple directly to other terms within the $(4 f)^{10}$ configuration. They correspond to a 
tensor operator with $l=2$, so they can couple the ground-state manifold both to $J=7$ and to $J=6$. The bare quadrupole interaction is determined by a single reduced matrix element, which can be defined by an arbitrary component of the quadrupole tensor. For example, in terms of the $m=+2$ operator component

$$
\hat{Q}_{2}=e \int d^{3} \mathbf{r} \hat{\psi}^{\dagger}(\mathbf{r}) r^{2} Y_{2}^{0}(\Omega) \hat{\psi}(\mathbf{r})
$$

(where $\Omega$ denotes the electron's angular coordinates), the reduced matrix element is

$$
\begin{aligned}
Q & =\left\langle J, J\left|\hat{Q}_{z z}\right| J, J\right\rangle=\sqrt{\frac{16 \pi}{5}}\left\langle Q_{2}\right\rangle \\
& =\left\langle J \| \hat{Q}_{2}|| J\right\rangle\left(\begin{array}{ccc}
J & J & 2 \\
-J & J & 0
\end{array}\right) .
\end{aligned}
$$

In our calculations, we determine $Q$ by fitting, though it could in principle also be determined from the form of the Ho orbitals. As shown below in Sec. III we find the value of $\mathbf{k} \cdot \mathbf{Q}$ is small (of order $10^{-15} \mathrm{~m}$ ).

Jorgensen and Judd [31] pointed out there is a second source of quadrupole coupling that can be much larger: electrical polarization of the surrounding atoms by the uniform field of a plane wave produces a potential at position $\mathbf{r}$ :

$$
\sum_{I} \frac{\alpha_{I} \mathcal{E} \cdot\left(\mathbf{r}-\mathbf{R}_{I}\right)}{4 \pi\left|\mathbf{r}-\mathbf{R}_{I}\right|^{4}}
$$

Because the environment lacks inversion symmetry, this potential can possess a quadrupole component at the Ho site. The resulting interaction, summed over electrons $i$, can be written [32] as $-\mathcal{E} \cdot \mathbf{D}_{\text {eff }}$, where the effective dipole contribution $D_{\text {Jeff }}$ has spherical tensor components

$$
D_{\mathrm{eff}}^{(1)}=\frac{\sqrt{35}}{4 \pi} \sum_{i, I} \frac{r_{i}^{2}}{R_{I}^{4}} \alpha_{I}\left(C_{i}^{(2)} \cdot C_{I}^{(3)}\right)^{(1)} .
$$

Because of the $R_{I}^{4}$ denominator this term converges rapidly with shells of neighboring ions. However because it is a spherical tensor of order 2 for the electrons, and order 3 for the ions, it is indistinguishable when fitting from the term with $\lambda=2, t=3$ in the Judd-Ofelt expansion [Eqs. (18) and (17)] below.

\section{Electric dipole terms}

The electric dipole operator can only couple states of opposite parity. Since the $S_{4}$ point group does not contain the inversion operation, parity is not a good quantum number and odd-parity atomic states can be mixed into the even-parity $(4 f)^{10}$ configuration by the odd-parity parts of the crystal field. However, such admixtures must involve higher-lying configurations such as $(4 f)^{9}(4 g)$ and $(4 f)^{9}(5 d)$, which have energies of more than $70000 \mathrm{~cm}^{-1}$ above the ground state. On the assumption that the crystal-field splittings of these excited configurations are negligible in comparison to their excitation energies, Judd [25] and Ofelt [26] showed that the matrix elements of the effective dipole operator can be written

$$
\left\langle\alpha\left|\hat{D}_{q}^{(1)}\right| \beta\right\rangle=\sum_{p t} \gamma_{t p} A_{t p},
$$

with

$$
\begin{aligned}
\gamma_{t p}= & \sum_{\lambda \text { even }}(2 \lambda+1)(-1)^{p+q}\left(\begin{array}{ccc}
1 & \lambda & t \\
q & -p-q & p
\end{array}\right) \\
& \times\left\langle\alpha\left|\hat{U}_{p+q}^{(\lambda)}\right| \beta\right\rangle \Xi(t, \lambda)
\end{aligned}
$$

and

$$
\begin{aligned}
\Xi(t, \lambda)= & 2 \sum_{n^{\prime}, l^{\prime}}(2 l+1)\left(2 l^{\prime}+1\right)(-1)^{l+l^{\prime}}\left\{\begin{array}{ccc}
1 & \lambda & t \\
l & l^{\prime} & l
\end{array}\right\} \\
& \times\left(\begin{array}{lll}
l & 1 & l^{\prime} \\
0 & 0 & 0
\end{array}\right)\left(\begin{array}{lll}
l^{\prime} & t & l \\
0 & 0 & 0
\end{array}\right) \frac{\left\langle n l|r| n^{\prime} l^{\prime}\right\rangle\left\langle n l\left|r^{t}\right| n^{\prime} l^{\prime}\right\rangle}{\Delta\left(n^{\prime}, l^{\prime}\right)},
\end{aligned}
$$

where $\hat{U}^{(\lambda)}$ is a tensor operator constructed from summing the unit one-electron tensor operator over all the $f$ electrons, i.e.,

$$
\hat{U}^{(\lambda)}=\sum_{i=1}^{N} \hat{u}^{(\lambda)}(i) \quad \text { with } \quad\left\langle n l\left\|\hat{u}^{(\lambda)}\right\| n^{\prime} l^{\prime}\right\rangle=\delta_{n, n^{\prime}} \delta_{l, l^{\prime}} .
$$

The crystal-field coefficients entering the sum in Eq. (17) are only those with odd $t$ (since contributions from even $t$ cancel; this is as expected since they conserve parity), while the sum over $\lambda$ in Eq. (18) goes over even $\lambda$ from 0 to 6 . The singleelectron quantum numbers $n^{\prime}$ and $l^{\prime}$ give the shell and angular momentum index of the excited (i.e., non- $f$-shell) electron. The single-electron matrix elements will be nonzero only if $l^{\prime}=l \pm 1$, i.e., for admixtures involving electrons excited to $d$ or $g$ states. For the $d$ states crystal-field coefficients up to $t=5$ are required; for $g$ states we need up to $t=7$.

In practice the crystal-field coefficients $A_{t p}$ are, like those with even $t$ which determine the structure within each manifold, difficult to obtain exactly; point-ion models give unsatisfactory results [27] and a fitting procedure is generally adopted. In previous literature $[16,17]$ only the intensities of entire absorption bands were used in this fitting; here we use our measured results to fit the absorption strengths of individual crystal-field lines. From this information we could in principle determine the individual $A_{t p}$, if data from a free ion were used to constrain the matrix elements and energy denominator in (19). The approach taken here is instead to fit the integrated intensities of all the electronic lines, under the assumption that only two configurations $\left[(4 f)^{9}(5 g)\right.$ and $(4 f)^{9}(5 d)$ ] contribute to Eq. (17). Apart from angular factors entirely determined by the angular momentum quantum numbers, these two contributions differ only through the final factors in Eq. (19), which depend on the value $t$ (but not on $\lambda$ ). The fit therefore determines ratios, relating the contributions from each configuration for $t=3$ and $t=5$ (only $g$ states contribute in the case of $t=7$ ).

A check on the validity of the Judd-Ofelt approach is provided by one previous calculation that went beyond it, making a direct calculation of the first-order wave functions mixed in by the odd-parity crystal-field coefficients [33], avoiding assumptions about the nature of the states mixed in or their exception energies. It concentrated on calculating the optical absorption at shorter wavelengths than measured here (between $300 \mathrm{~nm}$ and $1300 \mathrm{~nm}$ ). It demonstrated good agreement between theory and room-temperature experiments, and 
showed that higher-order contributions neglected in the JuddOfelt approach are small (around 3-4 orders of magnitude smaller than the lowest-order terms). One important difference with the calculations described here was the inclusion of odd-parity crystal-field parameters $B_{l}{ }^{m}$ with $l=1$; these terms are forbidden at low temperature in perfect $S_{4}$ symmetry but can arise as a result of ionic fluctuations at finite temperature.

\section{Combined interaction with radiation}

Accounting for all these interactions, the total effective pulse area within the rotating wave approximation for coherent excitation by the electric (dipole and quadrupole) and magnetic interactions is then

$$
\begin{aligned}
A_{\mathrm{eff}}= & A_{e}+A_{m}+A_{q} \\
= & \frac{\mu_{i j, e}}{\hbar} \cdot \int_{0}^{T} \mathbf{E}(t) d t+\frac{\mu_{i j, m}}{\hbar} \cdot \int_{0}^{T} \mathbf{B}(t) d t \\
& +\frac{1}{6 \hbar} \mathbf{k} \cdot \mathbf{Q} \cdot \int_{0}^{T} \mathbf{E}(t) d t \\
= & {\left[e\langle i|\mathbf{r}| j\rangle \cdot \mathbf{e}_{E}+\frac{n}{c} \frac{\mu_{B}\langle i|\mathbf{L}+2 \mathbf{S}| j\rangle}{\hbar} \cdot \mathbf{e}_{B}\right.} \\
& \left.+\frac{\omega n Q}{6 L(2 L-1) \hbar c} \hat{\mathbf{k}} \cdot\langle i|\hat{\mathbf{Q}}| j\rangle \cdot \mathbf{e}_{E}\right] \int_{0}^{T} \frac{E(t)}{\hbar} d t,
\end{aligned}
$$

where $\mathbf{e}_{E}$ and $\mathbf{e}_{B}$ are the (unit) polarization vectors for the electric and magnetic fields, respectively. The corresponding incoherent absorption rate within Fermi's golden rule is

$$
\begin{aligned}
K_{i j}= & \frac{2 \pi}{\hbar} \mid e\langle i|\mathbf{r}| j\rangle \cdot \mathbf{e}_{E}+\frac{n}{c} \frac{\mu_{B}\langle i|\mathbf{L}+2 \mathbf{S}| j\rangle}{\hbar} \cdot \mathbf{e}_{B} \\
& +\left.\frac{\omega n Q}{6 L(2 L-1) \hbar c} \hat{\mathbf{k}} \cdot\langle i|\hat{\mathbf{Q}}| j\rangle \cdot \mathbf{e}_{E}\right|^{2}\left|E\left(\omega_{i j}\right)\right|^{2} \\
\equiv & \frac{2 \pi}{\hbar}\left|M_{i j}\right|^{2}\left|E\left(\omega_{i j}\right)\right|^{2},
\end{aligned}
$$

where $E\left(\omega_{i j}\right)$ is the Fourier component of the electric field at the resonant frequency of the transition $i \rightarrow j$. For a given polarization and frequency we take Eq. (22) as the definition of a single effective matrix element $M_{i j}$ with the dimensions of an electric dipole, characterizing the overall coupling to the electric field. The Einstein $A$ and $B$ coefficients, effective oscillator strength $f$, and integrated cross section $\sigma$ for the absorption (all taken in free space) can then be expressed in terms of this quantity:

$$
\begin{aligned}
A_{i j} & =\frac{\omega_{i j}^{3}}{\pi \epsilon_{0} \hbar c^{3}}\left|M_{i j}\right|^{2}, \\
B_{i j} & =\frac{\pi}{\hbar^{2} \epsilon_{0}}\left|M_{i j}\right|^{2}, \\
f_{i j} & =\frac{2 m_{e} \omega_{i j}}{\hbar e^{2}}\left|M_{i j}\right|^{2}, \\
\sigma_{i j} & =\frac{\pi \omega_{i j}}{\hbar \epsilon_{0} c}\left|M_{i j}\right|^{2}=\frac{\pi^{2} c^{2}}{\omega_{i j}^{2}} A_{i j} \\
& =\frac{\hbar \omega_{i j}}{c} B_{i j}=\frac{\pi e^{2}}{2 \epsilon_{0} m_{e} c} f_{i j} .
\end{aligned}
$$

TABLE III. Number of electronic states of different symmetries present in each manifold. The symmetries are given both their standard names and the labels associated with the $\Gamma$ point in solid-state physics. Note the entry in the final column gives the number of degenerate $\Gamma_{34}$ pairs.

\begin{tabular}{lccc}
\hline \hline & \multicolumn{3}{c}{ Symmetry } \\
\cline { 2 - 4 }$J$ & $\Gamma_{1}$ & $\Gamma_{2}$ & $\Gamma_{34}$ \\
\hline 8 & 5 & 4 & 4 \\
7 & 3 & 4 & 4 \\
6 & 3 & 4 & 3 \\
5 & 3 & 2 & 3 \\
\hline \hline
\end{tabular}

The frequency-dependent cross section is in turn related to the attenuation coefficient $\alpha$ and the measured absorbance $\mathcal{A}$ through the relations

$$
\alpha(\omega)=\sigma(\omega) n_{\mathrm{Ho}}, \quad \mathcal{A}(\omega)=\frac{\alpha(\omega) \ell}{\ln (10)},
$$

where $n_{\mathrm{Ho}}$ is the volume density of Ho ions (assumed dilute so interactions can be neglected) and $\ell$ is the sample thickness.

\section{Numbers of lines in the light of selection rules}

The total number of lines expected in each manifold is fixed by symmetry. The $2 J+1$ electronic states in the ${ }^{5} L_{J}$ manifold are split into crystal-field eigenstates, each of which has the symmetry of an irreducible representation of the $S_{4}$ point group; the number of states of each type is shown in Table III. The lowest-energy $J=8$ manifold has a doubly degenerate $\Gamma_{34}$ state as the ground state, with a $\Gamma_{2}$ state lying $6.85 \mathrm{~cm}^{-1}$ above it. Thus at temperatures below about $5 \mathrm{~K}$ the majority of the population will be in the ground state and the absorption spectrum will be dominated by excitations from the ground state; as the temperature is raised the first new lines to appear should be satellites with frequencies $6.85 \mathrm{~cm}^{-1}$ below the ground-state absorptions, corresponding to excitations from the thermally populated $\Gamma_{2}$ state. The symmetry of singlet states is unambiguously determined by the polarization in which the satellite absorption is observed: for $\Gamma_{1}$ states the satellite is an electric-dipole transition seen when the light is in the $z$ polarization, whereas for $\Gamma_{2}$ it is a magnetic-dipole transition, observed when the light is $x$-polarized.

\section{E. Extraction of hyperfine parameters and line intensities from data}

The measured transition lines were fitted to a sum of eight Lorentzians in the form

$$
I(v)=a \cdot \sum_{i=1}^{8} \frac{\gamma / 2}{\left(v-v_{i}\right)^{2}+(\gamma / 2)^{2}}+b,
$$

where the fitting parameters are the amplitude $a$, the offset $b$, the linewidth $\gamma$, and the individual peak locations $v_{i}$. $\Delta v_{i} \equiv v_{i+1}-v_{i}$ were distributed with a linearly increasing or decreasing slope to account for higher-order hyperfine splittings, as described in Sec. II B 2. In the FIR region FabryPerot interference fringes were added into the fit, since they 
are prominent for these wavelengths owing to the decreased effect of the wedge and the higher refractive index below the reststrahlen band (3.08, compared to 1.45 at $1 \mu \mathrm{m})$.

The experimental line intensities were calculated by subtracting the fitted offset $b$ from a measured absorption line and numerically integrating. In cases of overlap between neighboring lines the non-overlapping parts were used to perform the Lorentzian fits and the extracted parameters were used to extrapolate the intensities in the overlapping region.

The relative signs of the hyperfine coupling coefficients for the $\Gamma_{34}$ states were determined by relating their respective hyperfine spacings to that of the ground state, keeping in mind that the observed spacing can either be a sum (for ED transitions) or a difference (for MD transitions) as described in Sec. II B 2.

The measured integrated absorbances were then fitted by an expression of the form

$$
\mathcal{A}_{i j}=\frac{\pi \omega_{i j} n_{\mathrm{Ho}} \ell}{\ln (10) \hbar \epsilon_{0} c}\left|M_{i j}\right|^{2}
$$

with

$$
\begin{aligned}
M_{i j}= & e\langle i| n^{3 / 2} b_{\mathrm{mag}} \hat{O}_{z}^{\mathrm{MD}}+n\left(\frac{n^{2}+2}{3}\right) b_{Q} \hat{O}_{x y}^{Q} \\
& +\sqrt{n}\left(\frac{n^{2}+2}{3}\right)\left(\sum_{t \in\{3,5\}} b_{t 2}\left[\hat{\gamma}_{t 2, x}^{\left(l^{\prime}=2\right)}+r_{t} \hat{\gamma}_{t 2, x}^{\left(l^{\prime}=4\right)}\right]\right. \\
& \left.+b_{72} \hat{\gamma}_{72, x}^{\left(l^{\prime}=4\right)}\right)|j\rangle
\end{aligned}
$$

for the $x$ (electric) polarization and

$$
\begin{aligned}
M_{i j}= & e\langle i| n^{3 / 2} b_{\text {mag }} \hat{O}_{x}^{\mathrm{MD}}+n\left(\frac{n^{2}+2}{3}\right) b_{Q} \hat{O}_{y z}^{Q} \\
& +\sqrt{n}\left(\frac{n^{2}+2}{3}\right)\left(\sum_{t \in\{3,5\}} b_{t 2}\left[\hat{\gamma}_{t 2, z}^{\left(l^{\prime}=2\right)}+r_{t} \hat{\gamma}_{t 2, z}^{\left(l^{\prime}=4\right)}\right]\right. \\
& \left.+b_{72} \hat{\gamma}_{72, z}^{\left(l^{\prime}=4\right)}\right)|j\rangle
\end{aligned}
$$

for the $z$ polarization. Here, the calculation of the $\hat{\gamma}$ operators is according to Eqs. (18) and (19), but excluding the unknown microscopic factors $\left\langle n l|r| n^{\prime} l^{\prime}\right\rangle\left\langle n l\left|r^{t}\right| n^{\prime} l^{\prime}\right\rangle / \Delta\left(n^{\prime}, l^{\prime}\right)$; the fitted crystal-field parameters therefore have the interpretation

$$
b_{t p}=\frac{\left\langle n l|r| n^{\prime} l^{\prime}\right\rangle\left\langle n l\left|r^{t}\right| n^{\prime} l^{\prime}\right\rangle}{\Delta\left(n^{\prime}, l^{\prime}\right)} A_{t p}
$$

with $l^{\prime}=2$ for $t=3,5$ and $l^{\prime}=4$ for $t=7$. The values of $\left|M_{i j}\right|^{2} / e^{2}$ define the corresponding effective line strengths, having dimensions of area. There are therefore nine optical fitting parameters: $b_{Q}, b_{t p}$ for $t \in\{3,5,7\}$ (which have dimensions of length) and $p= \pm 2$, and $r_{t}$ for $t \in\{3,5,7\}$ (which are dimensionless). As described above, the value of $b_{\text {mag }}=\frac{\mu_{B}}{e c}=\frac{\alpha}{2} a_{0}$ is determined from first principles and not fitted; furthermore the line intensities from the ${ }^{5} I_{8}$ manifold (where only incomplete experimental information is available owing to reststrahlen) and the ${ }^{5} F_{5}$ manifold (where there are some uncertainties in the line assignments) are not used to constrain the fit; however predicted intensities for the observed lines are shown in the accompanying tables. The refractive index $n$ was set equal to its measured values: $n=3.08$ at low frequencies (below the optic phonon band, i.e., for ${ }^{5} I_{8}$ ) and $n=1.45$ for high frequencies (above the optic phonon band for all other transitions). The weak anisotropy in $n$ in the birefringent crystal was neglected.

The occupancy of the first excited state, and hence the effective sample temperature, provides an additional fitting parameter when the excited-state image lines are included. This can be cross-checked with the relative intensity of the different hyperfine lines corresponding to each electronic transition to give two independent measurements of the electronic and nuclear temperature which can be compared with the nominal temperature of the bulk sample.

\section{RESULTS AND DISCUSSION}

\section{A. Optical absorption and assignment of transitions}

Figure 4 shows the polarization-resolved absorption spectra of five out of the six lowest-energy spin-orbit manifolds (the ${ }^{5} I_{4}$ manifold is not shown as the line strengths are very weak, within our measurement noise level) at a nominal temperature of $\sim 4 \mathrm{~K}$. The ${ }^{5} I_{8}$ manifold was measured with a $1 \% \mathrm{Ho}^{3+}$ sample, which gave a stronger signal; for the other manifolds a $0.3 \% \mathrm{Ho}^{3+}$ sample was used for better line resolution, but a few weak lines were visible only in the $1 \%$ sample. Additional lines beyond the expected $2 J+1$ in each manifold are visible. Many of these additional lines are $6.85 \mathrm{~cm}^{-1}$ below the ground-state transitions, as indicated by horizontal arrows in the figures. They originate in transitions from the first excited state in the ${ }^{5} I_{8}$ manifold, which has previously been measured with EPR [8], neutron scattering [2], and high-temperature optical spectroscopy [14]. Tables IV-VIII summarize the observed transition lines and their hyperfine splittings.

The absorptions of the ${ }^{5} I_{7},{ }^{5} I_{6}$, and ${ }^{5} I_{5}$ manifolds were used to fit the parameters appearing in the electric dipole matrix elements (31), and hence the odd-parity crystal-field terms. The absorption intensities from the first excited state were also computed; the fitted excited-state occupation probability $p$ was used to estimate the temperature. The global best fit was used both to calculate integrated intensities for comparison with experiment and to calculate the relevant electric or magnetic dipole matrix elements for each transition. For each transition these electric and magnetic dipole moments are given in their natural atomic units $e a_{0}$ and $\mu_{B}=\frac{\alpha}{2} e a_{0} c$, respectively; the global best-fit parameters are shown in Table IX. In all cases the electric dipole matrix elements should be interpreted as effective operators that also include the Judd-Jorgensen contributions to the quadrupolar interaction [32].

The main tool we use for line assignment is the hyperfine spacing, considering the symmetries of the initial and final state, the type of transition (ED or MD), the polarization in which the line was observed, and any other transitions to the same final state from initial states other than the ground state (in practice mainly 8.2). We adopt the same convention as Ref. [18], giving the average hyperfine spacing in each $\Gamma_{34}$ doublet the same sign as the expectation value $\left\langle\Gamma_{3}\left|\hat{J}_{z}\right| \Gamma_{3}\right\rangle$ 


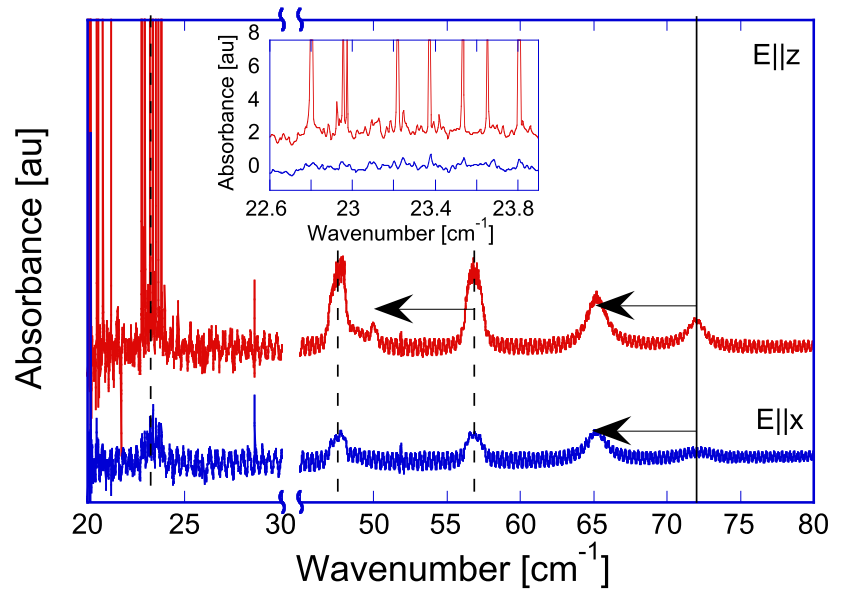

(a)

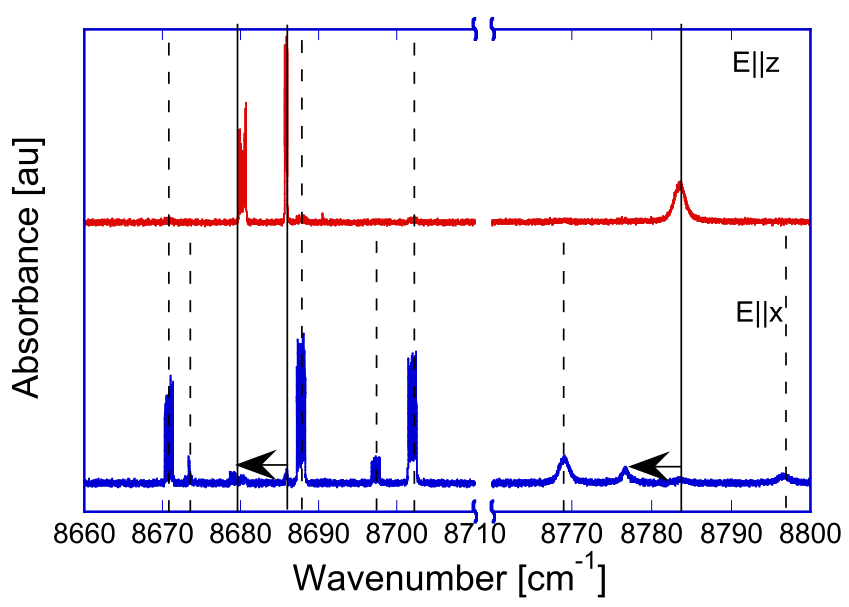

(c)

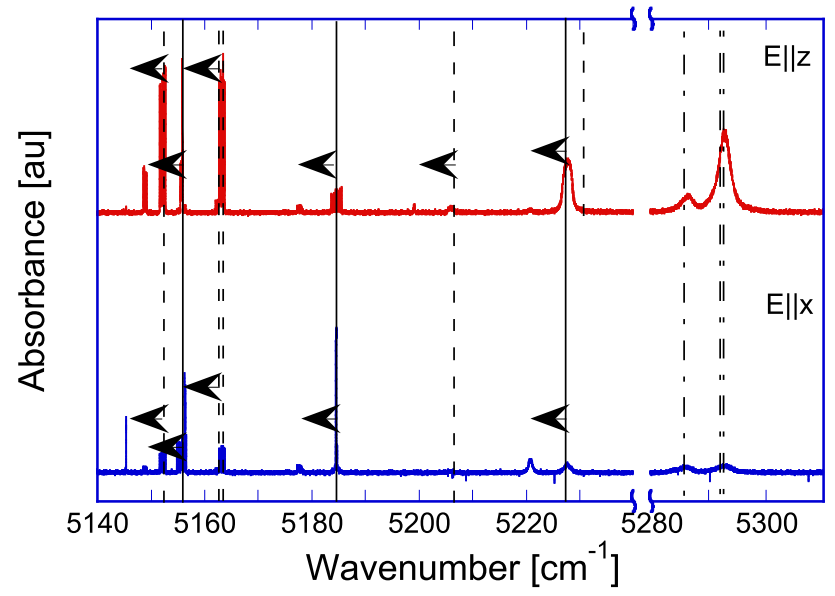

(b)

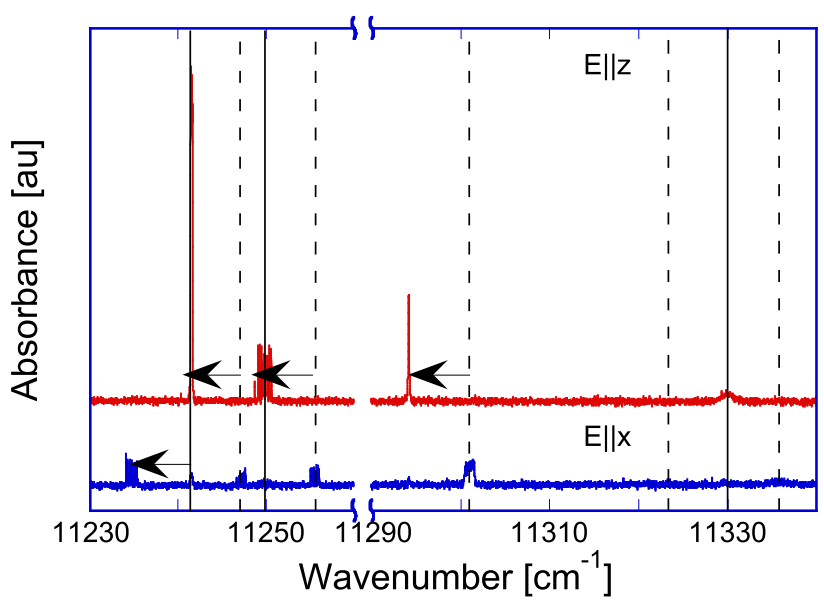

(d)

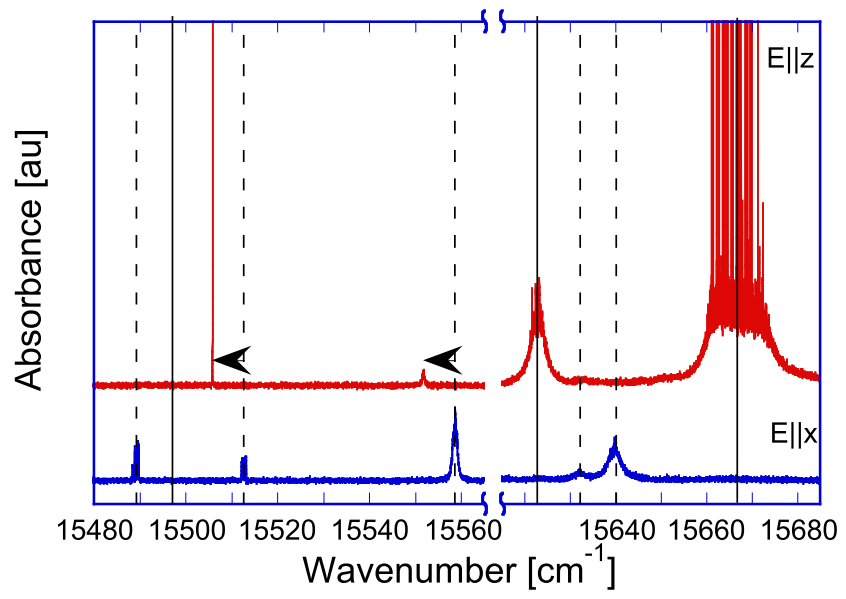

(e)

FIG. 4. Polarization-resolved $\mathrm{LiYF}_{4}: \mathrm{Ho}^{3+}$ optical absorbance at $4 \mathrm{~K}$. (a) ${ }^{5} I_{8}$. Inset is a blowup of the $8.1 \rightarrow 8.3$ transition. It differs from the spectrum in Fig. 6(a) as it is polarization resolved and most of the absorption is in the $z$ polarization (MD). (b) ${ }^{5} I_{7}$. (c) ${ }^{5} I_{6}$. (d) ${ }^{5} I_{5}$. (e) ${ }^{5} F_{5}$. The ${ }^{5} I_{4}$ manifold could not be observed. The different polarizations are offset for clarity. The dashed and solid lines are guides to the eye for transitions from the ground state to singlet and doublet states, respectively. The arrows pointing left connect ground-state (8.1) transitions with transitions from 8.2 that have the same upper state. The dash-dot lines in Fig. 4(b) are transitions whose symmetry could not be determined in this work. Phonon absorption bands [29] mask higher-lying ${ }^{5} I_{8}$ states and were not included in (a). Fabry-Perot fringes are visible in (a). The $\mathrm{Ho}^{3+}$ concentration is $1 \%$ (a), $0.3 \%$ in all other cases. 
TABLE IV. Energy states and the observed lines associated with them in the ${ }^{5} I_{8}$ manifold. $T=4.2 \mathrm{~K}, 1 \% \mathrm{Ho}^{3+}$. The line frequencies in the case of multiplets are the average position of the peaks. The experimental uncertainties are the $95 \%$ confidence margins of the fit; calculated intensities are based on the fits to the ${ }^{5} I_{7},{ }^{5} I_{6}$, and ${ }^{5} I_{5}$ absorptions.

\begin{tabular}{|c|c|c|c|c|c|c|c|c|c|c|c|c|}
\hline \multirow[b]{4}{*}{ Index } & \multirow{4}{*}{$\begin{array}{l}\text { Frequency } \\
\left(\mathrm{cm}^{-1}\right)\end{array}$} & \multirow{2}{*}{\multicolumn{3}{|c|}{ State }} & \multicolumn{8}{|c|}{ Associated observed transitions } \\
\hline & & & & & \multirow{3}{*}{$\begin{array}{l}\text { Initial } \\
\text { state }\end{array}$} & \multirow{3}{*}{$\begin{array}{l}\text { Frequency } \\
\left(\mathrm{cm}^{-1}\right)\end{array}$} & \multirow{3}{*}{$\begin{array}{l}\text { Polar- } \\
\text { ization }\end{array}$} & \multirow{3}{*}{$\begin{array}{l}\text { HFS } \\
\left(\mathrm{cm}^{-1}\right)\end{array}$} & \multirow[b]{3}{*}{ Type } & \multirow{2}{*}{\multicolumn{2}{|c|}{$\begin{array}{c}\text { Integrated } \\
\text { intensity }\left(\mathrm{cm}^{-1}\right)\end{array}$}} & \multirow{3}{*}{$\begin{array}{l}\text { Matrix element } \\
\quad \text { (calc) }\end{array}$} \\
\hline & & \multirow{2}{*}{$\begin{array}{l}\text { Sym- } \\
\text { metry }\end{array}$} & \multicolumn{2}{|c|}{ Avg HFS $\left(\mathrm{cm}^{-1}\right)$} & & & & & & & & \\
\hline & & & (meas) & (calc) & & & & & & (meas) & (calc) & \\
\hline 8.1 & 0 & $\Gamma_{34}$ & -0.146 & -0.131 & & & & & & & & \\
\hline 8.2 & 6.85 & $\Gamma_{2}$ & 0 & & & & & & & & 0.01 & $4.19 \mu_{B} ; 0.036 e a_{0}$ \\
\hline \multirow[t]{2}{*}{8.3} & 23.3 & $\Gamma_{2}$ & 0 & & 8.1 & $23.31 \pm 0.00$ & $x$ & $0.144 \pm 0.001$ & ED & $0.30 \pm 0.03$ & 0.02 & $0.023 e a_{0}$ \\
\hline & & & & & 8.1 & $23.30 \pm 0.00$ & $z$ & $0.143 \pm 0.001$ & MD & $0.99 \pm 0.20$ & 0.66 & $3.74 \mu_{B}$ \\
\hline \multirow[t]{2}{*}{8.4} & 47.6 & $\Gamma_{1}$ & 0 & & 8.1 & $47.60 \pm 0.01$ & $x$ & $0.144 \pm 0.002$ & ED & $0.55 \pm 0.02$ & 0.01 & $3.7 \times 10^{-3} e a_{0}$ \\
\hline & & & & & 8.1 & $47.61 \pm 0.01$ & $z$ & $0.139 \pm 0.003$ & MD & $1.61 \pm 0.02$ & 1.13 & $3.41 \mu_{B}$ \\
\hline \multirow[t]{3}{*}{8.5} & 56.9 & $\Gamma_{1}$ & 0 & & 8.1 & $56.92 \pm 0.01$ & $x$ & $0.145 \pm 0.002$ & $\mathrm{ED}$ & $0.61 \pm 0.14$ & 0.01 & $3.6 \times 10^{-3} e a_{0}$ \\
\hline & & & & & 8.1 & $56.92 \pm 0.01$ & $z$ & $0.146 \pm 0.002$ & MD & $1.79 \pm 0.04$ & 1.44 & $3.52 \mu_{B}$ \\
\hline & & & & & 8.2 & $50.06 \pm 0.00$ & $z$ & $0.000 \pm 4.218$ & $\mathrm{ED}$ & $0.10 \pm 0.03$ & 0.02 & $9.8 \times 10^{-3} e a_{0}$ \\
\hline \multirow[t]{4}{*}{8.6} & 71.9 & $\Gamma_{34}$ & 0.075 & 0.095 & 8.1 & $72.12 \pm 0.03$ & $x$ & $0.250 \pm 0.01$ & MD & $0.24 \pm 0.02$ & 0.04 & $0.55 \mu_{B}$ \\
\hline & & & & & 8.1 & $71.62 \pm 0.10$ & $z$ & $0.081 \pm 0.023$ & ED & $0.69 \pm 0.02$ & 0.087 & $0.023 e a_{0}$ \\
\hline & & & & & 8.2 & $65.36 \pm 0.09$ & $x$ & $0.094 \pm 0.025$ & ED & $0.88 \pm 0.04$ & 0.01 & $0.020 e a_{0}$ \\
\hline & & & & & 8.2 & $65.63 \pm 0.13$ & $z$ & $0.073 \pm 0.032$ & MD & $1.65 \pm 0.07$ & 0.37 & $4.21 \mu_{B}$ \\
\hline
\end{tabular}

in the component transforming as $M_{J}=+1$. Since $A_{J}<0$, an isolated state with positive hyperfine splitting has a lowest component in which a $\Gamma_{3}$ electronic part is paired with a $M_{I}=$ $+3 / 2$ nuclear state and a $\Gamma_{4}$ electronic part with a $M_{I}=-3 / 2$ nuclear state, and vice versa, although in practice some mixing may occur.

Our assignments are somewhat different from previous work. For example, examination of the polarization of transition lines leads to an opposite assignment of singlet symmetry $\left(\Gamma_{1} \leftrightarrow \Gamma_{2}\right)$ to that by Walsh et al. [17] (states 7.3, 7.4, and 7.6 in Table V), Karayianis et al. [13] (states 5.2 and 5.5 in Table VII), and Christensen [14] (states 7.3 and 7.4). By looking at the line shapes and hyperfine splittings we determine a different singlet/doublet symmetry (e.g., states 5.1-5.4 in [17]), or that the transitions are from the ground state and not from an excited state (states 7.3 and 6.2 in [13]). Generally, lines at the high-energy end of manifolds are broader and Lorentzian-shaped owing to lifetime broadening. This makes the hyperfine separation less obvious, as seen in states 7.9-7.11 in Table V. A direct measurement of these lifetimes will be presented in a future paper.

\section{The ${ }^{5} I_{8}$ manifold}

Figure 4(a) shows the transitions to the lower excited states of the ${ }^{5} I_{8}$ manifold, apart from the first excited state at $6.85 \mathrm{~cm}^{-1}$ which lies below the range of this experimental setup. The hyperfine-split line of the second excited state is magnified in the inset, and the eightfold split can be clearly seen. This line and its hyperfine splitting have been previously measured [30], but to our knowledge have not been published. The narrow feature at $51.9 \mathrm{~cm}^{-1}$ and another at $28.6 \mathrm{~cm}^{-1}$ are not assigned; the difference between them $\left(23.3 \mathrm{~cm}^{-1}\right)$ does however correspond to state 8.3. The asymmetric line shape in, e.g., state 8.4 is caused by the nonuniform spacing of the hyperfine coupling, as shown in Eq. (10).

\section{The ${ }^{5} I_{7}$ manifold}

The hyperfine spacings of the ${ }^{5} I_{7}$ states [see Fig. 4(b)] below $5230 \mathrm{~cm}^{-1}$ can be assigned unambiguously because the hyperfine spacings and selection rules are consistent with polarization, transition type, and the appearance of pairs of transitions from states 8.1 and 8.2 . Above $5230 \mathrm{~cm}^{-1}$ there is a weak singlet state at $5232 \mathrm{~cm}^{-1}$ that is observed only in the $1 \%$ sample; this leaves four states (a $\Gamma_{1}$ and a $\Gamma_{2}$ singlet, and a $\Gamma_{34}$ doublet) that must exist between 5286 and $5293 \mathrm{~cm}^{-1}$ but cannot be unambiguously resolved. The experimental spectrum shows two broad bands in each polarization centered around $5293 \mathrm{~cm}^{-1}$ and $5286 \mathrm{~cm}^{-1}$, respectively; because of the $\sim 7 \mathrm{~cm}^{-1}$ spacing between them we provisionally assign the band at $5293 \mathrm{~cm}^{-1}$ to three overlapping ground-state transitions, and the band at $5286 \mathrm{~cm}^{-1}$ to the corresponding satellite transitions from state 8.2.

\section{The ${ }^{5} I_{6}$ and ${ }^{5} I_{5}$ manifolds}

For $J \leqslant 6 \mathrm{MD}$ transitions are not allowed and the level assignment is generally simpler. There are three doublets expected in ${ }^{5} I_{6}$ and all three are clearly visible in the $z$ polarization, with the expected satellite absorptions from state 8.2 in the $x$ polarization [Fig. 4(c)]. The lower two (6.3 and $6.4)$ lie very close in energy, 6.3 having the smaller measured hyperfine splitting. Theoretically the states are also close but the upper state is predicted to have the smaller hyperfine splitting; we therefore assigned the upper state in the theory to transition 6.3.

In ${ }^{5} I_{5}$ most lines are weak [Fig. 4(d)], the absorption being dominated by two doublets, each of which is clearly visible in the $z$ polarization with the expected $x$-polarized satellite; a further (weaker) doublet lies near the top of the manifold. Three further $x$-polarized lines have $z$-polarized satellites, confirming them as $\Gamma_{1}$ states. 
TABLE V. Energy states and the associated lines in the ${ }^{5} I_{7}$ manifold. $T=3.8 \mathrm{~K}, 0.3 \% \mathrm{Ho}^{3+}$. Transitions $7.9-7.11$ cannot be individually assigned because the lines overlap and hyperfine splitting cannot be determined with confidence; the observed lines are therefore presumed to arise from a combination of electric and magnetic dipole transitions (denoted XD). Uncertainties in integrated intensities are quoted as $0.00[0](x)$ when they are less than $0.0[0] 1$. The dagger $(\dagger)$ indicates that the weak transition $8.1 \rightarrow 7.8$ was only observed in a sample containing $1 \% \mathrm{Ho}^{3+}$ and the integrated absorption was scaled accordingly. This notation applies to the tables below as well.

\begin{tabular}{|c|c|c|c|c|c|c|c|c|c|c|c|c|}
\hline \multirow[b]{4}{*}{ Index } & \multirow{4}{*}{$\begin{array}{l}\text { Frequency } \\
\left(\mathrm{cm}^{-1}\right)\end{array}$} & \multirow{2}{*}{\multicolumn{3}{|c|}{ State }} & \multicolumn{8}{|c|}{ Associated observed transitions } \\
\hline & & & & & \multirow{3}{*}{$\begin{array}{c}\text { Initial } \\
\text { state }\end{array}$} & \multirow{3}{*}{$\begin{array}{l}\text { Frequency } \\
\left(\mathrm{cm}^{-1}\right)\end{array}$} & \multirow{3}{*}{$\begin{array}{l}\text { Polar- } \\
\text { ization }\end{array}$} & \multirow{3}{*}{$\begin{array}{l}\mathrm{HFS} \\
\left(\mathrm{cm}^{-1}\right)\end{array}$} & \multirow[b]{3}{*}{ Type } & \multirow{2}{*}{\multicolumn{2}{|c|}{$\begin{array}{c}\text { Integrated } \\
\text { intensity }\left(\mathrm{cm}^{-1}\right)\end{array}$}} & \multirow{3}{*}{$\begin{array}{l}\text { Matrix element } \\
\quad \text { (calc) }\end{array}$} \\
\hline & & \multirow{2}{*}{$\begin{array}{l}\text { Sym- } \\
\text { metry }\end{array}$} & \multicolumn{2}{|c|}{ Avg HFS $\left(\mathrm{cm}^{-1}\right)$} & & & & & & & & \\
\hline & & & (meas) & (calc) & & & & & & (meas) & (calc) & \\
\hline \multirow[t]{4}{*}{7.1} & 5152.2 & $\Gamma_{2}$ & 0 & & 8.1 & $5152.23 \pm 0.00$ & $x$ & $0.146 \pm 0.000$ & $\mathrm{ED}$ & $0.07 \pm 0.00$ & 0.06 & $3.5 \times 10^{-4} e a_{0}$ \\
\hline & & & & & 8.1 & $5152.23 \pm 0.00$ & $z$ & $0.146 \pm 0.000$ & $\mathrm{MD}$ & $0.43 \pm 0.01$ & 0.63 & $0.73 \mu_{B}$ \\
\hline & & & & & 8.2 & $5145.39 \pm 0.00$ & $x$ & $0.007 \pm 0.000$ & MD & $0.03 \pm 0.00$ & 0.02 & $0.22 \mu_{B}$ \\
\hline & & & & & 8.3 & $5128.93 \pm 0.00$ & $x$ & $0.001 \pm 0.000$ & MD & $0.00(3) \pm 0.00$ & & $0.62 \mu_{B}$ \\
\hline \multirow[t]{5}{*}{7.2} & 5155.8 & $\Gamma_{34}$ & 0.089 & 0.104 & 8.1 & $5155.76 \pm 0.00$ & $x$ & $0.234 \pm 0.000$ & MD & $0.10 \pm 0.01$ & 0.07 & $0.17 \mu_{B}$ \\
\hline & & & & & 8.1 & $5155.76 \pm 0.00$ & $z$ & $0.058 \pm 0.000$ & $\mathrm{ED}$ & $0.11 \pm 0.01$ & 0.08 & $4.3 \times 10^{-4} e a_{0}$ \\
\hline & & & & & 8.2 & $5148.94 \pm 0.00$ & $x$ & $0.089 \pm 0.000$ & $\mathrm{ED}$ & $0.01 \pm 0.00$ & 0.003 & $1.7 \times 10^{-4} e a_{0}$ \\
\hline & & & & & 8.2 & $5148.94 \pm 0.00$ & $z$ & $0.089 \pm 0.000$ & MD & $0.10 \pm 0.01$ & 0.11 & $0.61 \mu_{B}$ \\
\hline & & & & & 8.3 & $5132.45 \pm 0.00$ & $z$ & $0.089 \pm 0.001$ & MD & $0.01 \pm 0.00$ & & $0.71 \mu_{B}$ \\
\hline \multirow[t]{4}{*}{7.3} & 5162.7 & $\Gamma_{1}$ & 0 & & 8.1 & $5162.71 \pm 0.01$ & $x$ & $0.151 \pm 0.001$ & $\mathrm{ED}$ & $0.02 \pm 0.00$ & 0.01 & $1.3 \times 10^{-4} e a_{0}$ \\
\hline & & & & & 8.1 & $5162.68 \pm 0.00$ & $z$ & $0.144 \pm 0.000$ & $\mathrm{MD}$ & $0.03 \pm 0.00$ & 0.02 & $0.13 \mu_{B}$ \\
\hline & & & & & 8.2 & $5156.43 \pm 0.00$ & $x$ & $0.010 \pm 0.001$ & MD & $0.05 \pm 0.10$ & 0.10 & \\
\hline & & & & & 8.2 & $5155.89 \pm 0.00$ & $z$ & $0.007 \pm 0.000$ & $\mathrm{ED}$ & $0.08 \pm 0.00$ & 0.04 & $5.3 \times 10^{-6} e a_{0}$ \\
\hline \multirow[t]{3}{*}{7.4} & 5163.3 & $\Gamma_{2}$ & 0 & & 8.1 & $5163.28 \pm 0.00$ & $x$ & $0.146 \pm 0.000$ & $\mathrm{ED}$ & $0.07 \pm 0.00$ & 0.08 & $4.1 \times 10^{-4} e a_{0}$ \\
\hline & & & & & 8.1 & $5163.28 \pm 0.00$ & $z$ & $0.146 \pm 0.00$ & $\mathrm{MD}$ & $0.41 \pm 0.02$ & 0.62 & $0.72 \mu_{B}$ \\
\hline & & & & & 8.2 & $5156.43 \pm 0.00$ & $x$ & $0.010 \pm 0.001$ & $\mathrm{MD}$ & $0.05 \pm 0.01$ & 0.05 & $0.58 \mu_{B}$ \\
\hline \multirow[t]{4}{*}{7.5} & 5184.7 & $\Gamma_{34}$ & -0.132 & -0.130 & 8.1 & $5184.66 \pm 0.00$ & $x$ & $0.019 \pm 0.000$ & $\mathrm{MD}$ & $0.18 \pm 0.01$ & 0.69 & $0.54 \mu_{B}$ \\
\hline & & & & & 8.1 & $5184.66 \pm 0.00$ & $z$ & $0.278 \pm 0.000$ & $\mathrm{ED}$ & $0.10 \pm 0.00$ & 0.12 & $3.4 \times 10^{-4} e a_{0}$ \\
\hline & & & & & 8.2 & $5177.84 \pm 0.00$ & $x$ & $0.132 \pm 0.000$ & $\mathrm{ED}$ & $0.03 \pm 0.00$ & 0.02 & $2.6 \times 10^{-4} e a_{0}$ \\
\hline & & & & & 8.2 & $5177.84 \pm 0.00$ & $z$ & $0.132 \pm 0.000$ & MD & $0.03 \pm 0.00$ & 0.03 & $0.31 \mu_{B}$ \\
\hline \multirow[t]{3}{*}{7.6} & 5206.0 & $\Gamma_{1}$ & 0 & & 8.1 & $5206.08 \pm 0.01$ & $x$ & $0.146 \pm 0.003$ & $\mathrm{ED}$ & $0.01 \pm 0.00$ & 0.13 & $1.8 \times 10^{-4} e a_{0}$ \\
\hline & & & & & 8.1 & $5206.02 \pm 0.01$ & $z$ & $0.150 \pm 0.002$ & MD & $0.04 \pm 0.00$ & 0.03 & $0.16 \mu_{B}$ \\
\hline & & & & & 8.2 & $5199.20 \pm 0.02$ & $z$ & $0.011 \pm 0.004$ & $\mathrm{ED}$ & $0.01 \pm 0.00$ & 0.05 & $5.8 \times 10^{-4} e a_{0}$ \\
\hline \multirow[t]{4}{*}{7.7} & 5228.0 & $\Gamma_{34}$ & -0.081 & -0.100 & 8.1 & $5228.06 \pm 0.89$ & $x$ & $0.076 \pm 0.187$ & MD & $0.11 \pm 0.01$ & 0.03 & $0.12 \mu_{B}$ \\
\hline & & & & & 8.1 & $5227.78 \pm 0.00$ & $z$ & $0.225 \pm 0.001$ & $\mathrm{ED}$ & $0.80 \pm 0.01$ & 0.55 & $4.9 \times 10^{-4} e a_{0}$ \\
\hline & & & & & 8.2 & $5220.93 \pm 0.02$ & $x$ & $0.080 \pm 0.006$ & $\mathrm{ED}$ & $0.11 \pm 0.00$ & 0.01 & $3.4 \times 10^{-5} e a_{0}$ \\
\hline & & & & & 8.2 & $5220.89 \pm 0.06$ & $z$ & $0.089 \pm 0.016$ & MD & $0.01 \pm 0.00$ & $0.00(4)$ & $0.115 \mu_{B}$ \\
\hline \multirow[t]{2}{*}{7.8} & 5232.2 & $\Gamma_{2}$ & 0 & & 8.1 & $5232.23 \pm 0.46$ & $x$ & $0.146 \pm 0.091$ & $\mathrm{ED}$ & $0.00(3) \pm 0.00(08)^{\dagger}$ & 0.08 & $3.3 \times 10^{-4} e a_{0}$ \\
\hline & & & & & 8.1 & $5232.23 \pm 34.42$ & $z$ & $0.045 \pm 6.769$ & MD & $0.01 \pm 0.00(06)^{\dagger}$ & 0.0002 & $0.013 \mu_{B}$ \\
\hline \multirow[t]{5}{*}{$7.9-7.11$} & $5286-5293$ & $\Gamma_{1}, \Gamma_{2}, \Gamma_{34}$ & & & 8.1 & $5292.32 \pm 0.13$ & $x$ & & $\mathrm{XD}$ & $0.17 \pm 0.01$ & 0.17 & $2.4 \times 10^{-4} e a_{0}\left(\Gamma_{2}\right)$ \\
\hline & & & & & & & & & & & & $\begin{array}{c}1.2 \times 10^{-4} e a_{0}\left(\Gamma_{1}\right) \\
0.19 \mu_{B}\left(\Gamma_{34}\right)\end{array}$ \\
\hline & & & & & 8.1 & $5292.71 \pm 0.02$ & $z$ & & $\mathrm{XD}$ & $1.98 \pm 0.01$ & 2.09 & $\begin{array}{c}9.0 \times 10^{-4} e a_{0}\left(\Gamma_{34}\right) \\
0.15 \mu_{B}\left(\Gamma_{2}\right)\end{array}$ \\
\hline & & & & & 8.2 & $5285.95 \pm 0.27$ & $x$ & & XD & $0.11 \pm 0.01$ & 0.16 & $0.077 \mu_{B}\left(\Gamma_{1}\right)$ \\
\hline & & & & & 8.2 & $5286.13 \pm 0.04$ & $z$ & & $\mathrm{XD}$ & $0.39 \pm 0.01$ & 0.35 & $1.6 \times 10^{-4} e a_{0}$ \\
\hline
\end{tabular}

\section{The ${ }^{5} F_{5}$ manifold}

$\operatorname{In}^{5} F_{5}$ there is a broad and very intense absorption band with $z$ polarization, centered around $15667 \mathrm{~cm}^{-1}$. We assign this to the highest $\Gamma_{34}$ doublet of the manifold, which is predicted to have an unusually strong dipole absorption. An additional strong absorption around $15622 \mathrm{~cm}^{-1}$ is associated with a second doublet state. Two $z$-polarized satellites to other $x$ polarized lines are observed, marking them as belonging to $\Gamma_{1}$ states, but the symmetries of the other singlets (including a strong $x$-polarized absorption near $15639 \mathrm{~cm}^{-1}$ ) are difficult to determine unambiguously. For this reason the theoretical results for this manifold are reported but not used in the fitting.

Figure 5 summarizes the intensity calculations by plotting predicted intensities against observed intensities for all lines. We find the correlation coefficients between the logarithms of the experimental and predicted intensities are 0.707 and 0.725 for the $x$-polarized and $z$-polarized transitions from the ground state, and 0.493 and 0.779 for the $x$ - and $z$-polarized transitions from the first excited state, respectively. Hence the predictions are more accurate for $z$ polarization then for $x$, and 
TABLE VI. Energy states and the observed lines associated with them in the ${ }^{5} I_{6}$ manifold. $T=3.8 \mathrm{~K}, 0.3 \% \mathrm{Ho}^{3+}$.

\begin{tabular}{|c|c|c|c|c|c|c|c|c|c|c|c|c|}
\hline & & & & & & & & Associated obser & ved tra & nsitions & & \\
\hline & & State & & & & & & & & I & & \\
\hline & & & Avg HFS & $\left(\mathrm{cm}^{-1}\right)$ & & & & & & intensity (c & $\left.m^{-1}\right)$ & Matrix \\
\hline Index & $\left(\mathrm{cm}^{-1}\right)$ & metry & (meas) & (calc) & state & $\left(\mathrm{cm}^{-1}\right)$ & ization & $\left(\mathrm{cm}^{-1}\right)$ & Type & (meas) & (calc) & $\left(e a_{0}\right)(\mathrm{calc})$ \\
\hline 6.1 & 8670.9 & $\Gamma_{2}$ & 0 & & 8.1 & $8670.89 \pm 0.00$ & $x$ & $0.146 \pm 0.000$ & ED & $0.25 \pm 0.01$ & 0.29 & $6.6 \times 10^{-5}$ \\
\hline 6.2 & 8673.4 & $\Gamma_{1}$ & 0 & & 8.1 & $8673.36 \pm 0.00$ & $x$ & $0.144 \pm 0.001$ & ED & $0.01 \pm 0.00$ & 0.14 & $1.1 \times 10^{-4}$ \\
\hline 6.3 & 8680.3 & $\Gamma_{34}$ & 0.021 & 0.049 & 8.1 & $8680.30 \pm 0.00$ & $z$ & $0.132 \pm 0.000$ & ED & $0.30 \pm 0.02$ & 0.09 & $1.5 \times 10^{-4}$ \\
\hline & & & & & 8.2 & $8673.50 \pm 0.01$ & $x$ & $0.021 \pm 0.001$ & ED & $0.02 \pm 0.00$ & 0.04 & $2.0 \times 10^{-4}$ \\
\hline 6.4 & 8685.9 & $\Gamma_{34}$ & 0.095 & 0.145 & 8.1 & $8685.89 \pm 0.00$ & $z$ & $0.050 \pm 0.000$ & ED & $0.33 \pm 0.02$ & 0.41 & $8.6 \times 10^{-5}$ \\
\hline & & & & & 8.2 & $8679.07 \pm 0.00$ & $x$ & $0.095 \pm 0.000$ & ED & $0.02 \pm 0.00$ & $0.00(5)$ & $3.6 \times 10^{-5}$ \\
\hline 6.5 & 8687.8 & $\Gamma_{2}$ & 0 & & 8.1 & $8687.79 \pm 0.00$ & $x$ & $0.146 \pm 0.000$ & ED & $0.60 \pm 0.03$ & 0.68 & $3.9 \times 10^{-5}$ \\
\hline 6.6 & 8697.4 & $\Gamma_{1}$ & 0 & & 8.1 & $8697.38 \pm 0.00$ & $x$ & $0.146 \pm 0.000$ & ED & $0.08 \pm 0.00$ & 0.03 & $1.9 \times 10^{-4}$ \\
\hline & & & & & 8.2 & $8690.59 \pm 0.01$ & $z$ & $0.012 \pm 0.001$ & ED & $0.00(4) \pm 0.00$ & $0.00(04)$ & $8.4 \times 10^{-5}$ \\
\hline 6.7 & 8702.1 & $\Gamma_{2}$ & 0 & & 8.1 & $8702.06 \pm 0.00$ & $x$ & $0.146 \pm 0.000$ & ED & $0.61 \pm 0.01$ & 0.38 & $8.7 \times 10^{-5}$ \\
\hline 6.8 & 8769.0 & $\Gamma_{1}$ & 0 & & 8.1 & $8769.05 \pm 0.02$ & $x$ & $0.145 \pm 0.006$ & $\mathrm{ED}$ & $0.36 \pm 0.01$ & 0.42 & $2.7 \times 10^{-4}$ \\
\hline 6.9 & 8783.6 & $\Gamma_{34}$ & $-0.095^{\dagger}$ & -0.007 & 8.1 & $8783.61 \pm 0.01$ & $z$ & $0.240 \pm 0.003$ & ED & $0.62 \pm 0.01$ & 0.40 & $1.5 \times 10^{-4}$ \\
\hline & & & & & 8.2 & $8776.84 \pm 0.04$ & $x$ & $0.095 \pm 0.01$ & ED & $0.13 \pm 0.01$ & 0.12 & $3.3 \times 10^{-4}$ \\
\hline 6.10 & 8796.5 & $\Gamma_{2}$ & 0 & & 8.1 & $8796.55 \pm 0.11$ & $x$ & $0.157 \pm 0.03$ & ED & $0.13 \pm 0.01$ & 0.01 & $1.1 \times 10^{-4}$ \\
\hline
\end{tabular}

more accurate for ground-state transitions then for excitedstate ones. In total these correlations are calculated from 61 lines (34 in the $x$ polarization and 27 in $z$ polarization), of which 31 were used in the fitting of the seven $b_{t p}$ and $r_{t}$ parameters; at least for the ground state, this can be regarded as satisfactory agreement. The fit quality is approximately uniform across the range of all five manifolds and for both electric and magnetic dipole transitions.

\section{B. Temperature dependence}

The temperature dependence of the linewidths was measured using the $0.3 \%$ concentration sample, and the results for the ${ }^{5} I_{8}$ manifold appear in Fig. 6 . The hyperfine splitting of state 8.2 is still visible at $16 \mathrm{~K}$ [Fig. 6(a)], and there is no obvious temperature broadening. The octets at $47 \mathrm{~cm}^{-1}$ and $57 \mathrm{~cm}^{-1}$ (8.4 and 8.5 , respectively) are still clearly visible at $24 \mathrm{~K}$, with no signs of broadening [Fig. 6(b)].

This temperature independence of the linewidth at low temperature is illustrated in Fig. 7(a), which shows two adjacent transition lines in the ${ }^{5} I_{7}$ manifold. The hyperfine peaks remain resolved up to $T \approx 25 \mathrm{~K}$. The linewidth can be fitted up to higher temperatures, and the results for lowest-energy singlet lines in each of the first three excited manifolds $(7.1,6.1$, and 5.2) in the $x$ polarization are shown in Fig. 7(b). The low-temperature FWHM is similar in each

TABLE VII. Energy states and the observed lines associated with them in the ${ }^{5} I_{5}$ manifold. $T=4.4 \mathrm{~K}, 0.3 \%$ Ho ${ }^{3+}$. The double dagger $(\dagger \dagger$ ) indicates that for state 5.4 , the line shape at $0.3 \%$ was noisy and so the line shape from a $1 \%$ sample was used instead.

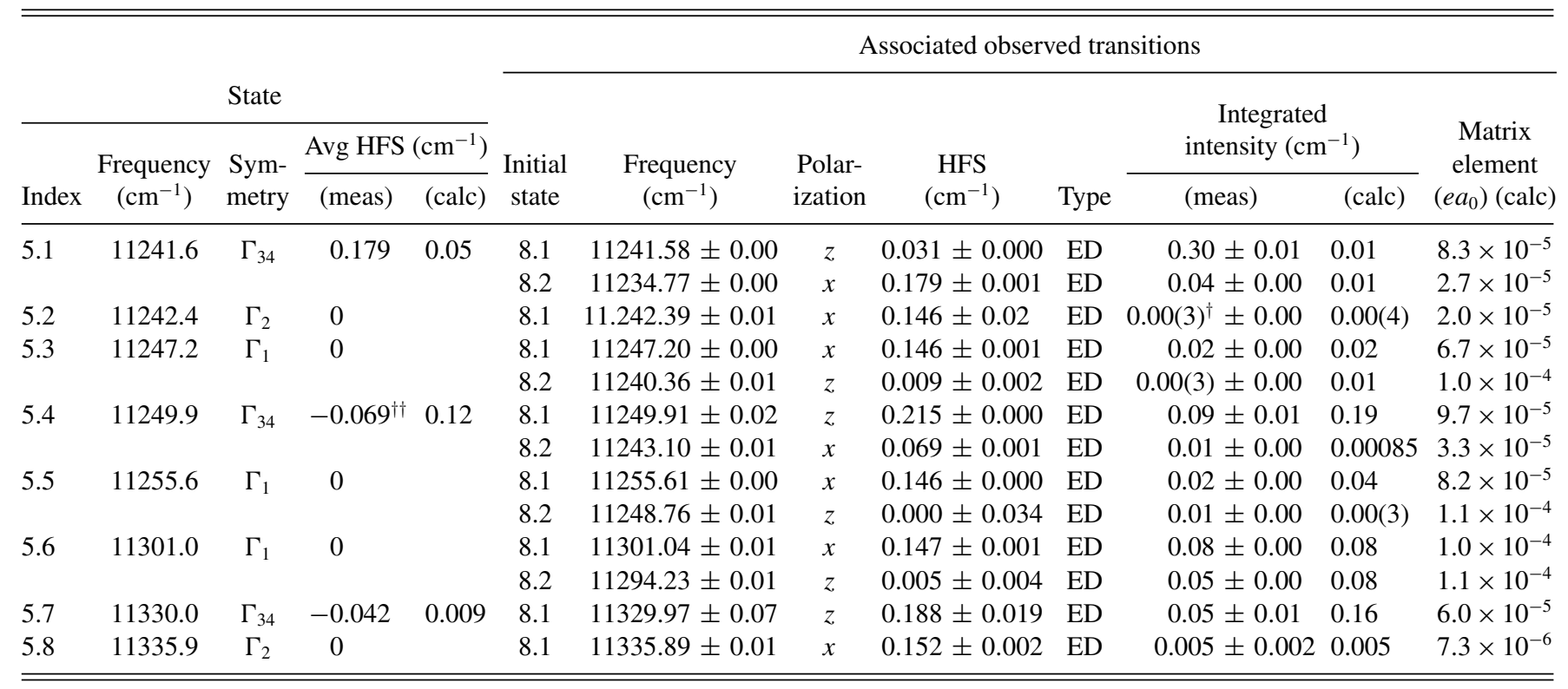


TABLE VIII. Observed transition lines and hyperfine splittings in the ${ }^{5} F_{5}$ manifold. $T=4.4 \mathrm{~K}, 0.3 \% \mathrm{Ho}^{3+}$.

\begin{tabular}{|c|c|c|c|c|c|c|c|c|c|c|c|c|}
\hline \multirow[b]{4}{*}{ Index } & \multirow{4}{*}{$\begin{array}{c}\text { Frequency } \\
\left(\mathrm{cm}^{-1}\right)\end{array}$} & \multirow{2}{*}{\multicolumn{3}{|c|}{ State }} & \multicolumn{8}{|c|}{ Associated observed transitions } \\
\hline & & & & & \multirow{3}{*}{$\begin{array}{l}\text { Initial } \\
\text { state }\end{array}$} & \multirow{3}{*}{$\begin{array}{l}\text { Frequency } \\
\left(\mathrm{cm}^{-1}\right)\end{array}$} & \multirow{3}{*}{$\begin{array}{l}\text { Polar- } \\
\text { ization }\end{array}$} & \multirow{3}{*}{$\begin{array}{l}\text { HFS } \\
\left(\mathrm{cm}^{-1}\right)\end{array}$} & \multirow[b]{3}{*}{ Type } & \multirow{2}{*}{\multicolumn{2}{|c|}{$\begin{array}{c}\text { Integrated } \\
\text { intensity }\left(\mathrm{cm}^{-1}\right)\end{array}$}} & \multirow{3}{*}{$\begin{array}{c}\text { Matrix } \\
\text { element } \\
\left(e a_{0}\right)(\text { calc })\end{array}$} \\
\hline & & \multirow{2}{*}{$\begin{array}{l}\text { Sym- } \\
\text { metry }\end{array}$} & \multicolumn{2}{|c|}{ Avg HFS $\left(\mathrm{cm}^{-1}\right)$} & & & & & & & & \\
\hline & & & (meas) & (calc) & & & & & & (meas) & (calc) & \\
\hline F5.1 & 15489.4 & $\Gamma_{2}$ & 0 & & 8.1 & $15489.39 \pm 0.00$ & $x$ & $0.146 \pm 0.000$ & ED & $0.20 \pm 0.01$ & 0.24 & $5.9 \times 10^{-5}$ \\
\hline \multirow[t]{2}{*}{ F5.2 } & 15495.4 & $\Gamma_{34}$ & -0.034 & 0.0068 & 8.1 & $15495.38 \pm 0.01$ & $z$ & $0.181 \pm 0.002$ & ED & $0.02 \pm 0.01$ & 0.85 & $2.1 \times 10^{-5}$ \\
\hline & & & & & 8.2 & $15488.56 \pm 0.01$ & $x$ & $0.034 \pm 0.001$ & ED & $0.04 \pm 0.01$ & 0.09 & $1.5 \times 10^{-4}$ \\
\hline \multirow[t]{2}{*}{ F5.3 } & 15512.7 & $\Gamma_{1}$ & 0 & & 8.1 & $15512.74 \pm 0.00$ & $x$ & $0.146 \pm 0.000$ & ED & $0.20 \pm 0.01$ & 0.91 & $2.1 \times 10^{-4}$ \\
\hline & & & & & 8.2 & $15505.92 \pm 0.00$ & $z$ & $0.000 \pm 224.090$ & ED & $1.53 \pm 0.04$ & 0.08 & $9.8 \times 10^{-5}$ \\
\hline \multirow[t]{2}{*}{ F5.4 } & 15558.7 & $\Gamma_{1}$ & 0 & & 8.1 & $15558.69 \pm 0.01$ & $x$ & $0.145 \pm 0.003$ & ED & $1.30 \pm 0.03$ & 0.13 & $1.1 \times 10^{-4}$ \\
\hline & & & & & 8.2 & $15551.73 \pm 1.03$ & $z$ & $0.025 \pm 0.267$ & $\mathrm{ED}$ & $0.16 \pm 0.01$ & 0.19 & $4.0 \times 10^{-6}$ \\
\hline F5.5 & 15622.8 & $\Gamma_{34}$ & -0.175 & 0.037 & 8.1 & $15622.84 \pm 0.09$ & $z$ & $0.320 \pm 0.025$ & ED & $5.32 \pm 0.14$ & 1.91 & $8.1 \times 10^{-5}$ \\
\hline F5.6 & 15632.1 & $\Gamma_{2}$ & 0 & & 8.1 & $15632.09 \pm 147.56$ & $x$ & $0.000 \pm 447.952$ & ED & $0.50 \pm 0.08$ & 0.20 & $5.4 \times 10^{-5}$ \\
\hline F5.7 & 15639.4 & $\Gamma_{1}$ & 0 & & 8.1 & $15639.43 \pm 0.95$ & $x$ & $0.140 \pm 0.197$ & ED & $2.27 \pm 0.04$ & 1.12 & $2.6 \times 10^{-4}$ \\
\hline F5.8 & 15667.1 & $\Gamma_{34}$ & $?$ & 0.085 & 8.1 & $15667.13 \pm 0.15$ & $z$ & $1.685 \pm 0.04$ & $\mathrm{ED}$ & $23.46 \pm 0.95$ & 36.90 & $9.8 \times 10^{-4}$ \\
\hline
\end{tabular}

case, being $\sim 0.04 \mathrm{~cm}^{-1}$ for ${ }^{5} I_{7}$ and ${ }^{5} I_{5}$ and $\sim 0.07 \mathrm{~cm}^{-1}$ for ${ }^{5} I_{6}$ (exceeding the experimental resolution of $\sim 0.01 \mathrm{~cm}^{-1}$ ); none of these numbers is limited by the optical lifetimes, direct measurements of which we will present in a future publication. However the linewidths for all three manifolds share a similar temperature dependence, which is well described by an Arrhenius fit with an activation energy $105 \mathrm{~K}<\Delta E / k_{B}<$ $131 \mathrm{~K}$. This suggests a common dephasing mechanism for all three lines, presumably involving the ground state; the activation energy corresponds approximately to the frequency of zone-center phonons, with Raman peaks observed from around $150 \mathrm{~cm}^{-1}$ in $\mathrm{LiHoF}_{4}$ [28,29].

The corresponding quality factors are $1.3 \times 10^{5}, 1.2 \times 10^{5}$, and $2.8 \times 10^{5}$ for $7.1,6.1$, and 5.2, respectively, making these transitions and several others (for example $8.1 \rightarrow$ F5.1, which reaches $Q=4.7 \times 10^{5}$ ) good candidates for highly selective coherent excitation. The upper state can also be a doublet with hyperfine splitting; because the hyperfine linewidths are much narrower than the hyperfine splittings they allow the possibility to pump individual electronuclear populations selectively. In the case of an upper-state singlet the hyperfine resolution

TABLE IX. Best-fit parameters for integrated absorbances according to Eqs. (31) and (32).

\begin{tabular}{lc}
\hline \hline Parameter & Value \\
\hline$b_{\text {mag }}$ & $0.0852 \mathrm{pm}$ \\
$b_{Q}$ & $0.0000 \mathrm{pm}$ \\
$b_{32 c}$ & $-1.480 \mathrm{pm}$ \\
$b_{32 s}$ & $1.148 \mathrm{pm}$ \\
$b_{52 c}$ & $0.284 \mathrm{pm}$ \\
$b_{52 s}$ & $-0.751 \mathrm{pm}$ \\
$b_{72 c}$ & $0.016 \mathrm{pm}$ \\
$b_{72 s}$ & $-0.022 \mathrm{pm}$ \\
$r_{3}$ & -1.469 \\
$r_{5}$ & -4.365 \\
$p$ & 0.257 \\
$T$ & $11.3 \mathrm{~K}$ \\
\hline \hline
\end{tabular}

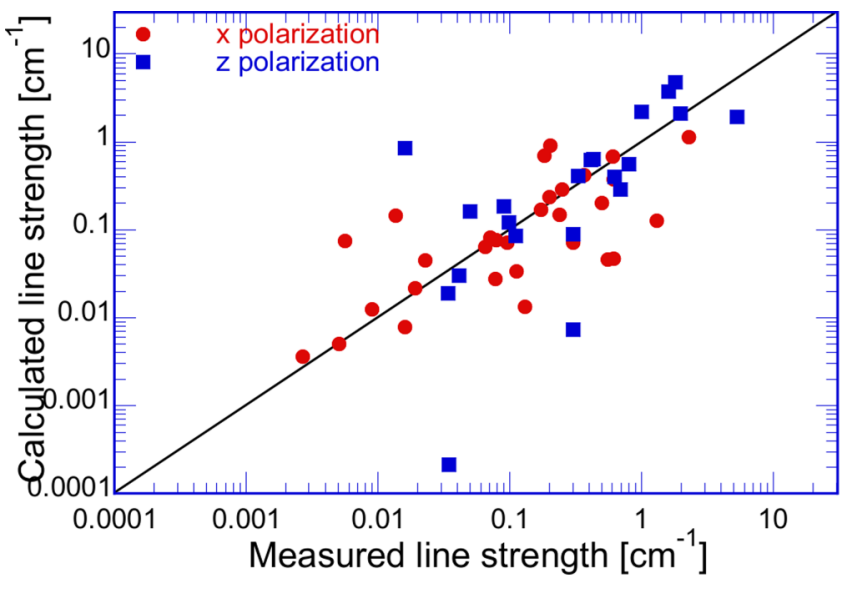

(a)

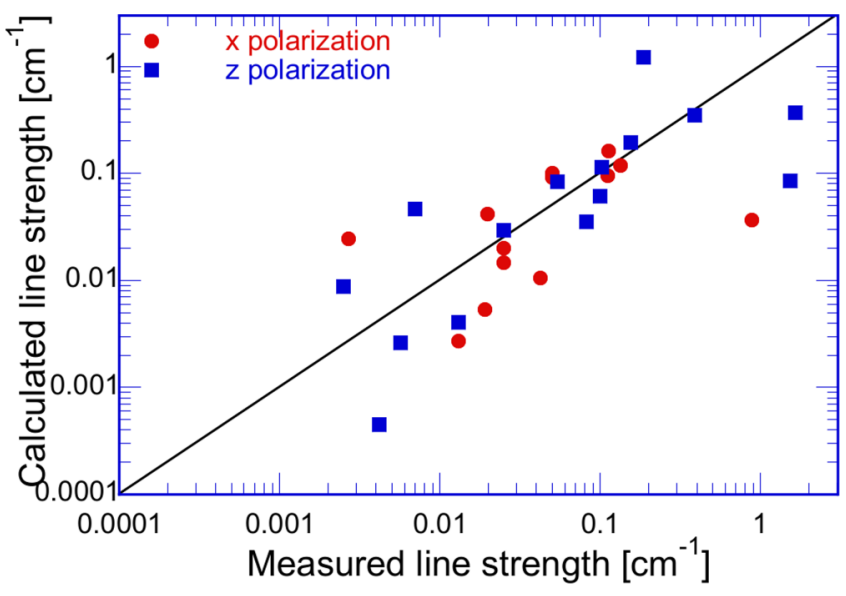

(b)

FIG. 5. Comparison of calculated and measured integrated intensities (including the ${ }^{5} I_{8}$ and ${ }^{5} F_{5}$ manifolds not used in the fitting). Panels (a) and (b) directly compare the measured and calculated intensities for transitions from the ground and first excited state, respectively. The red circles and blue squares are the $x$ and $z$ polarizations, respectively. The black $X=Y$ line is a guide to the eye. 


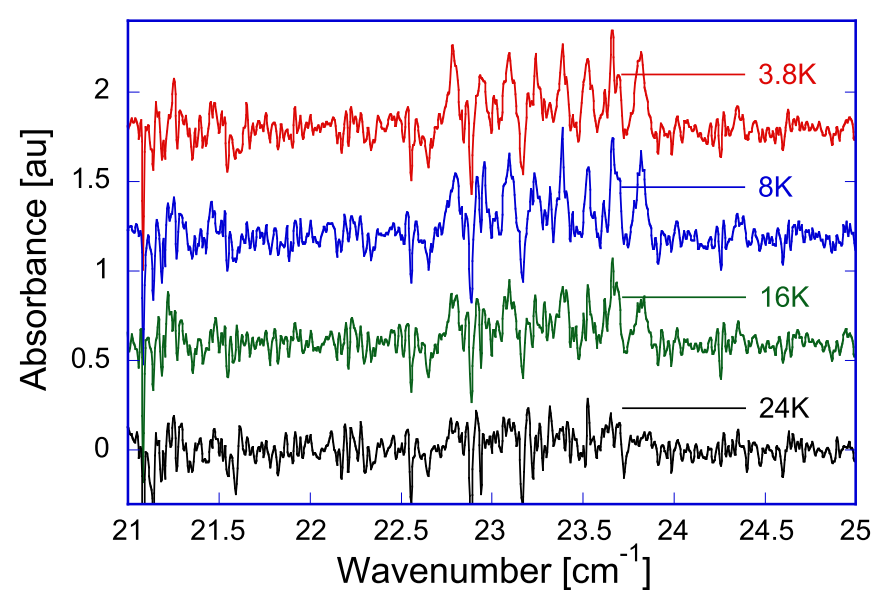

(a)

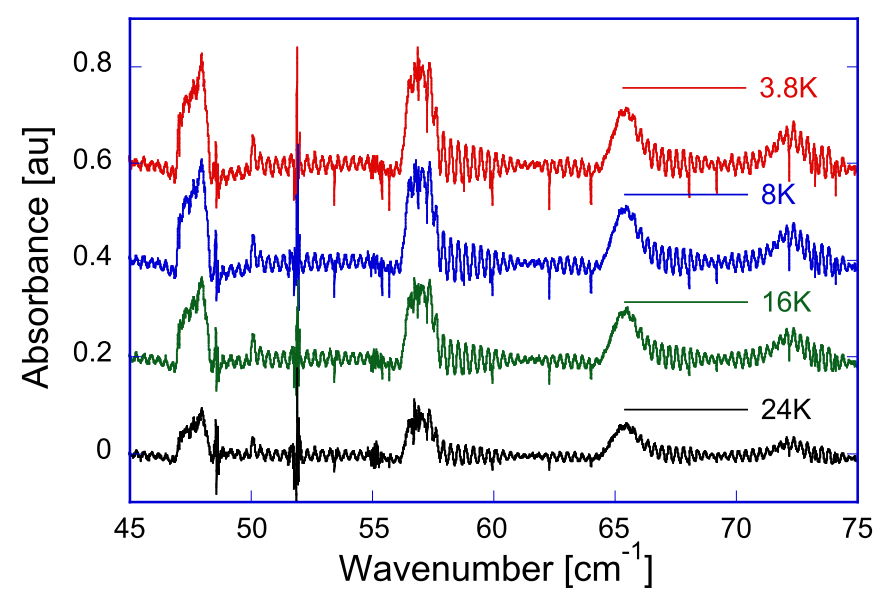

(b)

FIG. 6. Temperature dependence of transition line shapes within the ground-state manifold. The reference for these absorbance spectra is the spectrum at $32 \mathrm{~K}$, where these lines are no longer seen. The $\mathrm{Ho}^{3+}$ concentration is $1 \%$. The source light in this measurement is unpolarized to achieve maximum source intensity.

is lost, but the excited state is nonmagnetic; this opens up the possibility of switching off the electronic moment during the excitation.

\section{Relative contributions of magnetic and electric dipole transitions}

The fact that all the effective electric dipole lengths in Table IX are small $\left(\ll a_{0}\right)$ is a reflection of the weakness of the breaking of the parity selection rule; it is for this reason that the electric and magnetic dipole absorptions in ${ }^{5} I_{8}$ and ${ }^{5} I_{7}$ have comparable magnitudes. The effective quadrupole length $b_{Q}$ is found to be very small. The fits show a very small contribution of the magnetic quadrupole interaction, but similar magnetic and electric dipole coupling for the ${ }^{5} I_{7}$ to ${ }^{5} I_{8}$ transitions. Transitions to manifolds with $J \leqslant 6$ are almost entirely electric dipole in nature.

\section{CONCLUSIONS}

We have revisited the crystal-field lines of $\mathrm{LiYF}_{4}: \mathrm{Ho}^{3+}$ using high-resolution polarization-resolved absorption spec-

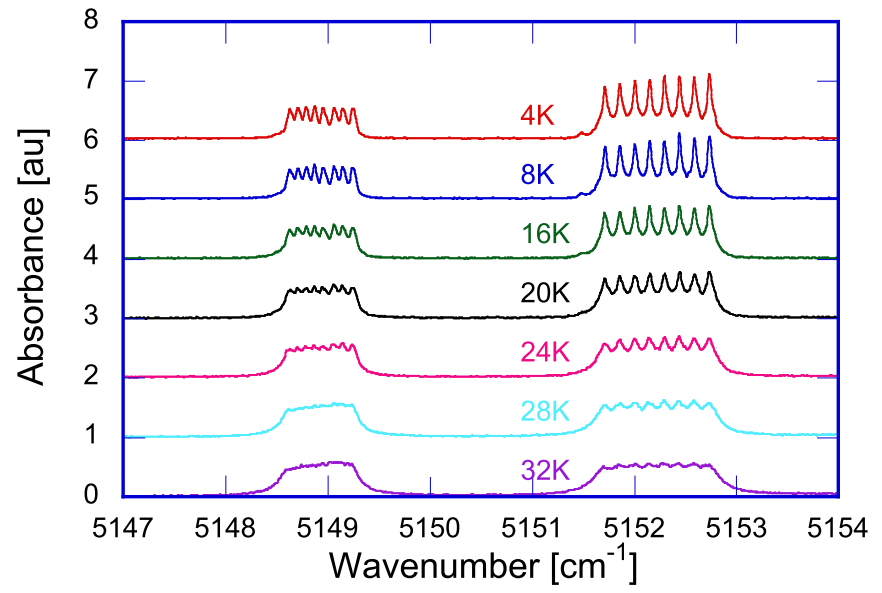

(a)

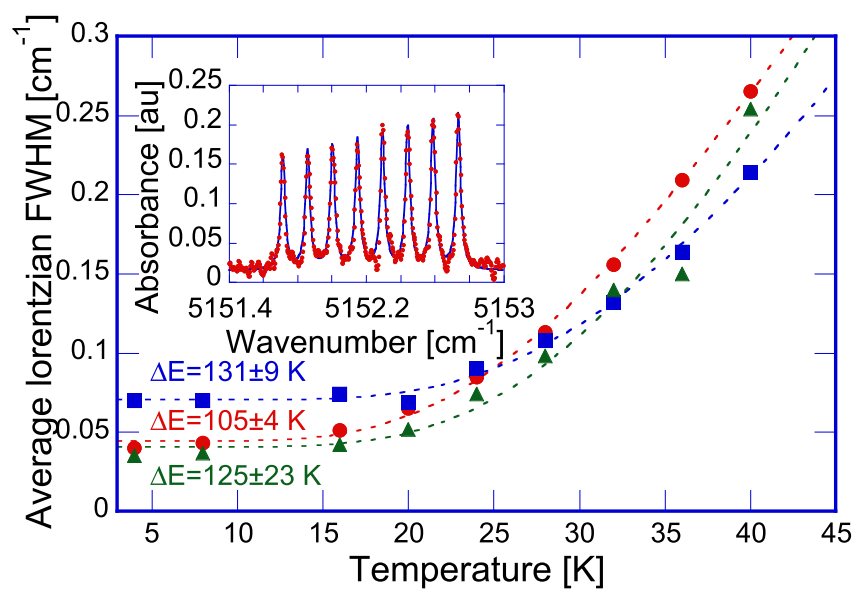

(b)

FIG. 7. Temperature broadening of the hyperfine line shapes. (a) Two hyperfine-split lines in the ${ }^{5} I_{7}$ manifold. (b) The Lorentzian hyperfine linewidths for transitions from the ground state to lowestenergy singlet states in the ${ }^{5} I_{7}$ (red circles), ${ }^{5} I_{6}$ (blue squares), and ${ }^{5} I_{5}$ (green triangles) manifolds, fitted to an Arrhenius equation. Inset is a typical multi-Lorentzian fit used to extract the width. Including a temperature-dependent occupancy of the ground-state hyperfine split (see Sec. II E) yields a nuclear temperature of $4.6 \pm 0.7 \mathrm{~K}$. The $\mathrm{Ho}^{3+}$ concentration is $0.3 \%$.

troscopy. Using the selection rules and the magnitudes of the hyperfine couplings we have assigned symmetry states to all but a few bands at the high-energy side of the ${ }^{5} I_{7}$ manifold. We have demonstrated that several previous assignments $[13,14,17]$ are inconsistent with either polarizations, hyperfine coupling, or both. In particular the assignment of doublets is clear but the assignment to some of the singlets is problematic. A major contribution to this difficulty is the broadening of states at the high-energy side of each manifold, which prevents the determination of the existence and magnitude of hyperfine coupling. This broadening is consistent with time-resolved photoluminescence spectra, which only show optical emission from the bottom of manifolds; the assumption is that rapid nonradiative decay occurs within each manifold.

Hence we can rigorously identify and assign almost all of the transitions using a combination of selection rules, hyperfine couplings, and intensities, which we fit with a limited number 
of parameters corresponding to the odd-parity crystal-field interactions. The main assumption in our global model is that two configurations, one with a $d$ electron $(l=3)$ and one with a $g$ electron $(l=5)$, dominate the mixing of odd-parity states which make electric dipole transitions weakly allowed; a further assumption is that the crystal-field splittings in these excited configurations are negligible compared to their excitation energies, allowing us to apply the Judd-Ofelt picture $[25,26]$. The remaining deviations from our fit are likely indications that one or the other of these assumptions is only partially satisfied.

For our low-temperature measurements, the absorption is dominated by transitions where the lowest state is the ground state. However there is a nonzero population in the lowestlying crystal-field excited state (8.2). We can use the ratio of the absorptions from this state and the ground state as a thermometer to determine the spin temperature of the sample; this also means that the absorption intensity is significantly redistributed compared to high-temperature measurements, where the thermal average effectively corresponds to an average over the entire ${ }^{5} I_{8}$ manifold $[16,17]$. Under those circumstances the absorption intensities in the Judd-Ofelt picture depend only on a weighted sum of the odd-parity crystal-field parameters: the electric-dipole absorption strength from a manifold of states with angular momentum quantum numbers $\{S, L, J\}$ to one with $\left\{S^{\prime}, L^{\prime}, J^{\prime}\right\}$ can be written as:

$$
\begin{aligned}
S_{E D}\left(J, J^{\prime}\right) & =\frac{1}{e^{2}} \sum_{M_{J}, M_{J^{\prime}}}\left|\left\langle S, L, J ; M_{J}\left|\hat{D}_{q}^{(1)}\right| S^{\prime}, L^{\prime}, J^{\prime} ; M_{J^{\prime}}\right\rangle\right|^{2} \\
& =\sum_{\lambda} \Omega_{\lambda}\left|\left\langle S, L, J \| \hat{U}^{(\lambda)}|| S^{\prime}, L^{\prime}, J^{\prime}\right\rangle\right|^{2}
\end{aligned}
$$

where $\hat{U}^{(\lambda)}$ is defined as in Eq. (20) and

$$
\Omega_{\lambda}=\frac{(2 \lambda+1)}{(2 J+1) e^{2}} \sum_{t} \frac{\Xi^{2}(t, \lambda)}{(2 t+1)} \sum_{p}\left|A_{t p}\right|^{2} .
$$

Hence only the combinations $\sum_{p}\left|A_{t p}\right|^{2}$ could be determined from previous fits. By contrast, the fits to our low-temperature data enable us to determine the individual coefficients $b_{t p}$ (which are proportional to $A_{t p}$ ) and also, by comparison with the known magnetic dipole terms, to measure the absolute matrix elements between individual crystal-field states.

In the case of $\mathrm{LiHo}_{x} \mathrm{Y}_{1-x} \mathrm{~F}_{4}$ this raises particularly interesting possibilities, since the symmetry group $S_{4}$ contains both one-dimensional and two-dimensional irreducible representations. This has the obvious consequence that the electronuclear qubits associated with the (electronic) doublet ground state can be read out using optical transitions to electronic singlet states at convenient mid- and near-infrared wavelengths. In addition, by exciting the system it is possible to switch between magnetic and nonmagnetic states in a way that would be impossible for a Kramers ion, where all states are twofold degenerate in zero field by time-reversal symmetry; our study quantifies the electromagnetic couplings needed to make this transition. The most promising excitations to observe this switching would be the long-lived states at the bottom of excited-state manifolds; for example, state 7.1 is a clearly resolved nonmagnetic state and can be pumped from one component of the ground-state doublet by either $x$-polarized light (via an electric dipole matrix element $3.7 \times 10^{-4} e a_{0}$ ) or $z$-polarized light (via its magnetic dipole matrix element $0.73 \mu_{B}$ ). In our quest for a workable qubit system we hope to next demonstrate coherent control of such a transition, and follow that by exciting and deexciting single-dopant and structured samples.

\section{ACKNOWLEDGMENTS}

The work at Caltech was supported by US Department of Energy Basic Energy Sciences Award No. DE-SC0014866. Work at UCL was supported by the Engineering and Physical Sciences Research Council (EPSRC) under Grants No. EP/H026622/1 and No. EP/M009564/1.
[1] H. M. Rønnow, R. Parthasarathy, J. Jensen, G. Aeppli, T. F. Rosenbaum, and D. F. McMorrow, Quantum phase transition of a magnet in a spin bath, Science 308, 389 (2005).

[2] H. M. Rønnow, J. Jensen, R. Parthasarathy, G. Aeppli, T. F. Rosenbaum, D. F. McMorrow, and C. Kraemer, Magnetic excitations near the quantum phase transition in the Ising ferromagnet $\mathrm{LiHoF}_{4}$, Phys. Rev. B 75, 054426 (2007).

[3] J. Brooke, D. Bitko, T. F. Rosenbaum, and G. Aeppli, Quantum annealing of a disordered magnet, Science 284, 779 (1999).

[4] M. A. Schmidt, D. M. Silevitch, G. Aeppli, and T. F. Rosenbaum, Using thermal boundary conditions to engineer the quantum state of a bulk magnet, Proc. Natl. Acad. Sci. USA 111, 3689 (2014).

[5] S. Ghosh, R. Parthasarathy, T. F. Rosenbaum, and G. Aeppli, Coherent spin oscillations in a disordered magnet, Science 296, 2195 (2002).

[6] S. Ghosh, T. F. Rosenbaum, G. Aeppli, and S. N. Coppersmith, Entangled quantum state of magnetic dipoles, Nature (London) 425, 48 (2003).
[7] C. Ancona-Torres, D. M. Silevitch, G. Aeppli, and T. F. Rosenbaum, Quantum and Classical Glass Transitions in $\mathrm{LiHo}_{x} \mathrm{Y}_{1-x} \mathrm{~F}_{4}$, Phys. Rev. Lett. 101, 057201 (2008).

[8] J. Magariño, J. Tuchendler, P. Beauvillain, and I. Laursen, EPR experiments in $\mathrm{LiTbF}_{4}, \mathrm{LiHoF}_{4}$, and $\mathrm{LiErF}_{4}$ at submillimeter frequencies, Phys. Rev. B 21, 18 (1980).

[9] P. A. Budni, M. L. Lemons, C. A. Miller, P. A. Ketteridge, L. A. Pomeranz, T. M. Pollak, P. G. Schunemann, K. L. Lanier, J. R. Mosto, and E. P. Chicklis, High power 1.9 micron pumped solid-state holmium lasers, in Pacific Rim Conference on Lasers and Electro-Optics, CLEO-Technical Digest (IEEE-Lasers and Electro-Optics Society, 2000), p. 564.

[10] E. P. Chicklis, C. S. Naiman, R. C. Folweiler, D. R. Gabbe, H. P. Jenssen, and A. Linz, High-efficiency room-temperature 2.06- $\mu \mathrm{m}$ laser using sensitized $\mathrm{Ho}^{3+}$ :YLF, Appl. Phys. Lett. 19, 119 (1971).

[11] C. Li, Y. Hang, X. Zhang, F. Zeng, T. Mauro, and J. Liu, Spectral properties of Tm, Ho:LiYF 4 laser crystal, J. Rare Earths 29, 592 (2011). 
[12] D. K. Young, J. A. Gupta, E. Johnston-Halperin, R. Epstein, Y. Kato, and D. D. Awschalom, Optical, electrical and magnetic manipulation of spins in semiconductors, Semicond. Sci. Technol. 17, 275 (2002).

[13] N. Karayianis, D. E. Wortman, and H. P. Jenssen, Analysis of the optical spectrum of $\mathrm{Ho}^{3+}$ in $\mathrm{LiYF}_{4}$, J. Phys. Chem. Solids 37, 675 (1976).

[14] H. P. Christensen, Spectroscopic analysis of $\mathrm{LiHoF}_{4}$ and $\mathrm{LiErF}_{4}$, Phys. Rev. B 19, 6564 (1979).

[15] J. Magariño, J. Tuchendler, J. P. D’Haenens, and A. Linz, Submillimeter resonance spectroscopy of $\mathrm{Ho}^{3+}$ in lithium yttrium fluoride, Phys. Rev. B 13, 2805 (1976).

[16] B. M. Walsh, N. P. Barnes, and B. Di Bartolo, Branching ratios, cross sections, and radiative lifetimes of rare earth ions in solids: Application to $\mathrm{Tm}^{3+}$ and $\mathrm{Ho}^{3+}$ ions in $\mathrm{LiYF}_{4}$, J. Appl. Phys. 83, 2772 (1998).

[17] B. M. Walsh, G. W. Grew, and N. P. Barnes, Energy levels and intensity parameters of $\mathrm{Ho}^{3+}$ ions in $\mathrm{GdLiF}_{4}, \mathrm{YLiF}_{4}$ and $\mathrm{LuLiF}_{4}$, J. Phys.: Condens. Matter 17, 7643 (2005).

[18] N. I. Agladze and M. N. Popova, Hyperfine structure in optical spectra of $\mathrm{LiYF}_{4}$-Ho, Solid State Commun. 55, 1097 (1985).

[19] N. I. Agladze, M. N. Popova, G. N. Zhizhin, V. J. Egorov, and M. A. Petrova, Isotope Structure in Optical Spectra of $\mathrm{LiYF}_{4}: \mathrm{Ho}^{3+}$, Phys. Rev. Lett. 66, 477 (1991).

[20] D. S. Pytalev, S. A. Klimin, and M. N. Popova, High-resolution optical study of $\mathrm{Ho}^{3+} \mathrm{Ho}^{3+}$ pairs in $\mathrm{LiY}_{1-x} \mathrm{Ho}_{x} \mathrm{~F}_{4}$ crystals, Phys. Lett. A 372, 2332 (2008).

[21] C. M. S. Gannarelli, D. M. Silevitch, T. F. Rosenbaum, G. Aeppl, and A. J. Fisher, Contribution of spin pairs to the magnetic response in a dilute dipolar ferromagnet, Phys. Rev. B 86, 014420 (2012).
[22] K. W. H. Stevens, Matrix elements and operator equivalents connected with the magnetic properties of rare earth ions, Proc. Phys. Soc. London, Sect. A 65, 209 (1952).

[23] M. T. Hutchings, Point-charge calculations of energy levels of magnetic ions in crystalline electric fields, Solid State Phys. 16, 227 (1964).

[24] R. Giraud, W. Wernsdorfer, A. M. Tkachuk, D. Mailly, and B. Barbara, Nuclear Spin Driven Quantum Relaxation in $\mathrm{LiY}_{0.998} \mathrm{Ho}_{0.002} \mathrm{~F}_{4}$, Phys. Rev. Lett. 87, 057203 (2001).

[25] B. R. Judd, Absorption intensities of rare-earth ions, Phys. Rev. 127, 750 (1962).

[26] G. S. Ofelt, Intensities of crystal spectra of rare-earth ions, J. Chem. Phys. 37, 511 (1962).

[27] D. Garcia, M. Faucher, and O. L. Malta, Electrostatic crystalfield contributions in rare-earth compounds with consistent multipolar effects. II. Contribution to $k$-odd parameters (transition probabilities), Phys. Rev. B 27, 7386 (1983).

[28] S. A. Miller, H. E. Rast, and H. H. Caspers, Lattice vibrations of $\mathrm{LiYF}_{4}$, J. Chem. Phys. 52, 4172 (1970).

[29] S. Salaün, M. T. Fornoni, A. Bulou, M. Rousseau, P. Simon, and J. Y. Gesland, Lattice dynamics of fluoride scheelites. I. Raman and infrared study of $\mathrm{LiYF}_{4}$ and $\mathrm{LiLnF}_{4}(\mathrm{Ln}=\mathrm{Ho}, \mathrm{Er}$, Tm and Yb), J. Phys.: Condens. Matter 9, 6941 (1997).

[30] N. I. Agladze, Ph.D. thesis, Institute of Spectroscopy, Russian Academy of Sciences, 1991.

[31] C. K. Jørgensen and B. R. Judd, Hypersensitive pseudoquadrupole transitions in lanthanides, Mol. Phys. 8, 281 (1964).

[32] B. R. Judd, Ionic transitions hypersensitive to environment, J. Chem. Phys. 70, 4830 (1979).

[33] Daniel Åberg and Sverker Edvardsson, Direct calculation of optical absorption amplitudes for trivalent rare-earth ions in $\mathrm{LiYF}_{4}$, Phys. Rev. B 65, 045111 (2002). 